\title{
Dynamic fracture analysis of functionally graded materials using ordinary state-based peridynamics
}

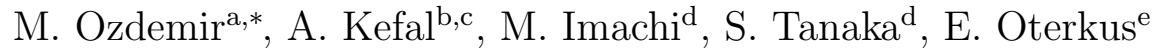 \\ ${ }^{a}$ Department of Naval Architecture and Marine Engineering, Ordu University, Ordu, \\ Turkey,e-mail:muratozdemir@odu.edu.tr \\ ${ }^{b}$ Faculty of Naval Architecture and Ocean Engineering, Istanbul Technical University, \\ Istanbul,Turkey, e-mail:kefaladnan@itu.edu.tr \\ ${ }^{c}$ Integrated Manufacturing Technologies Research and Application Center, Sabanci \\ University, Istanbul, Turkey, e-mail:adnankefal@sabanciuniv.edu \\ ${ }^{d}$ Graduate School of Engineering, Hiroshima University, Hiroshima, Japan, \\ e-mail:d174722@hiroshima-u.ac.jp, satoyuki@hiroshima-u.ac.jp \\ ${ }^{e}$ Department of Naval Architecture, Ocean and Marine Engineering, University of \\ Strathclyde, Glasgow, United Kingdom, e-mail:erkan.oterkus@strath.ac.uk
}

\begin{abstract}
Functionally graded materials are regarded as a special kind of composites capable of eliminating material interfaces and the delamination problems. Stress discontinuity can be avoided owing to smooth composition of the functionally graded ingredients. In this study, a recently emerged effective nonlocal continuum theory for solving fracture problems in solids and structures, peridynamics, is employed to simulate dynamic wave propagation as well as crack propagation in functionally graded materials. Specifically, the ordinary state-based formulation is adopted. The ordinary state-based formulation is slightly modified for the modelling of functionally graded materials. The averaging technique is employed to determine peridynamic parameters associated with the material properties. Firstly, a benchmark problem is considered to validate the present implementation of ordinary state-based peridynamics for brittle fracture of homogeneous materials. Then, the wave propagation in the functionally graded materials under impact loading is simulated. Finally, dynamic crack propagation in the functionally graded materials is studied. The evaluated crack paths and the displacement waves are compared with reference works including numerical and experimental results. Good agree-
\end{abstract}

\footnotetext{
${ }^{*}$ Corresponding author
} 
ment between the reference and present results is achieved. It is shown that a simple modification of ordinary state-based formulation has led to simulate dynamic fracture of functionally graded materials.

Keywords: peridynamics, dynamic fracture, functionally graded materials, crack

\section{Introduction}

Advanced manufacturing technologies enable to produce special purpose materials to meet system safety and structural performance requirements. Functionally Graded Materials (FGMs) can be considered as a solution to prevent stress singularities and material interfaces. Moreover, this kind of materials are capable of withstanding against simultaneous thermal and mechanical loads. Such favours of FGMs attract special interests among the researchers; Thai and Kim [1] has carried out a detailed literature survey on the theoretical works dealing with modelling and analysis of FGMs. FGMs may be sometimes found in military applications and the shock absorbing characteristics of FGMs are required to be investigated. Berezovski et al. [2] and Hedayatrasa et al. [3] accordingly examined the elastic wave propagation in FGM plates.

Cracks and evolution of damage/fracture have crucial influence on the structural integrity. A few decades ago, Eischen [4] proposed a quasi-path independent integral for the calculation of stress intensity factors (SIFs) for cracked solids assuming variable material properties. Abanto-Bueno and Lambros [5] carried out experiments on the FGMs for horizontal and slanted cracks adopting different gradation types. In addition, Kirugulige and Tippur $[6,7]$ investigated mixed-mode dynamic fracture of FGMs by conducting both experiments and cohesive zone Finite Element Method (FEM) simulations. Two crack configurations: (i) a crack on the stiff side of the specimen and (ii) a crack on the compliant side, were analysed by Kirugulige and Tippur [6, 7]. Kidane and co-workers [8,9] examined the mixed-mode crack propagation of FGMs under thermo-mechanical loads employing the asymptotic analysis method in conjunction with the displacement potentials. Recently, Doan et al. [10] and Hirshikesh et al. [11] have carried out an extensive work for the simulation of dynamic crack propagation in FGM plates utilizing the phase field modelling.

So far, the aforementioned numerical works in the previous paragraphs 
are mostly based on classical continuum theory and a special treatment is necessary for introducing cracks and evaluating fracture/damage patterns. As an alternative to classical continuum mechanics based approaches, Silling [12] presented a new robust numerical technique based on non-local continuum formulation, namely, peridynamics (PD). Reformulation of the equation of motion by PD inherently avoids spatial derivatives, therefore the discontinuities such as cracks can be treated in a straightforward manner. The simplest form of the PD can be considered as the bond-based formulation [13]. In this formulation, the volume dilation is not taken into account, therefore restrictions on the material properties arise in the bond-based PD formulation. Silling et al. [14] then proposed state-based PD formulation, which eliminates the restrictions on the material properties and allows the compressibility. Thanks to the advantage of PD for the modelling of discontinuities, one can find several research works dealing with fracture of the homogeneous materials [15-22] and FGMs [23-26]. Ha and Bobaru [15, 16] examined dynamic fracture of brittle materials employing the bond-based PD and the crack branching was also addressed in those works. Dipasquale et al. [18] discussed three different failure criteria for the Ordinary StateBased peridynamics (OSB-PD), and presented estimated fracture patterns by these criteria under mixed mode fracture condition. Imachi et al. [19, 20] obtained dynamic stress intensity factors (DSIFs) for stationary and rapidly growing cracks employing OSB-PD. Moreover, a numerical transition bond concept was proposed to suppress the oscillations in DSIFs [20]. Basoglu et al. [22] developed a computational model for the bond-based PD analysis of macro-crack and micro-cracked materials. Influence of different micro-crack arrangements on the propagation of macro-crack was examined.

Cheng et al. [23] investigated the dynamic wave propagation and fracture of FGMs by bond-based PD assuming several loading scenarios and compared the crack path estimations with the available experimental results. Then, Cheng and co-workers extended their work for the definition of bond properties in FGMs and composite weighted bond concept was proposed in Refs. [25, 26]. Liao et al. [27] modified the traditional state-based PD formulation for heat conduction in FGMs with cracks. Temperature distributions were obtained for stationary and growing cracks; however, the uniform crack growth rate was enforced rather than employing a failure criterion [27]. Detailed formulation of PD theory and its numerical implementation were extensively discussed and presented in the textbook of Madenci and Oterkus [28]. A recent detailed literature survey of PD theory was given by Ref. [29]. 
Even though the PD works are relatively common for laminated composites, e.g., see Refs. [30-33], the available literature for the FGMs is quite rare and these works are mainly carried out by using bond-based PD theory [23-26]. However, in the present work, two dimensional (2D) OSB-PD formulation [34] is employed for the accurate simulation of elastic wave propagation and crack propagation in FGMs. In this regard, a minor modification is conducted in the 2D OSB-PD formulation to effectively simulate variation of the material properties in FGMs. To the authors' knowledge, this work would be the first to employ 2D OSB-PD for the dynamic fracture analysis of FGMs in a detailed manner. We have previously implemented 2D OSB-PD formulation for evaluating DSIFs for stationary cracks [19], rapidly growing cracks [20] and crack arrest problems [21].

In the present work, a well-known benchmark problem for the dynamic brittle fracture of a homogeneous plate is considered first. It is known that dynamic crack propagation is associated with the elastic wave propagation under impact loading conditions. Therefore, the elastic wave propagation in FGMs is obtained by 2D OSB-PD formulation. Once the wave propagation is accurately simulated, we proceed for dynamic crack propagation in FGMs. For the comparison purposes of the present numerical results, we refer to available numerical and experimental results in the literature.

\section{Fundamentals of OSB-PD formulation}

PD theory is established based on non-local form of elasticity and it includes a length scale parameter called as horizon that makes the PD theory suitable for problems with different length scales $[12,14]$. Here, the 2D OSBPD formulation based on Ref. [34] is briefly revisited.

\subsection{Basic formulation}

In the PD modelling, the interaction between material points (particles) is defined by bonds. The material points exert PD forces through these bonds. The bonds are defined at the initial geometry, while the force states are determined in the deformed configuration as shown in Fig. 1. The given figure depicts the basic kinematics of material points in OSB-PD framework. Initial position vectors for a point of interest and its neighbouring particles are represented by $\boldsymbol{x}$ and $\boldsymbol{x}^{\prime}$, respectively. These points experience displacements and the final positions in the deformed state are denoted by $\boldsymbol{y}$ and $\boldsymbol{y}^{\prime}$. Relative position vector at the initial state is represented by $\boldsymbol{\xi}=\boldsymbol{x}^{\prime}-\boldsymbol{x}$. 


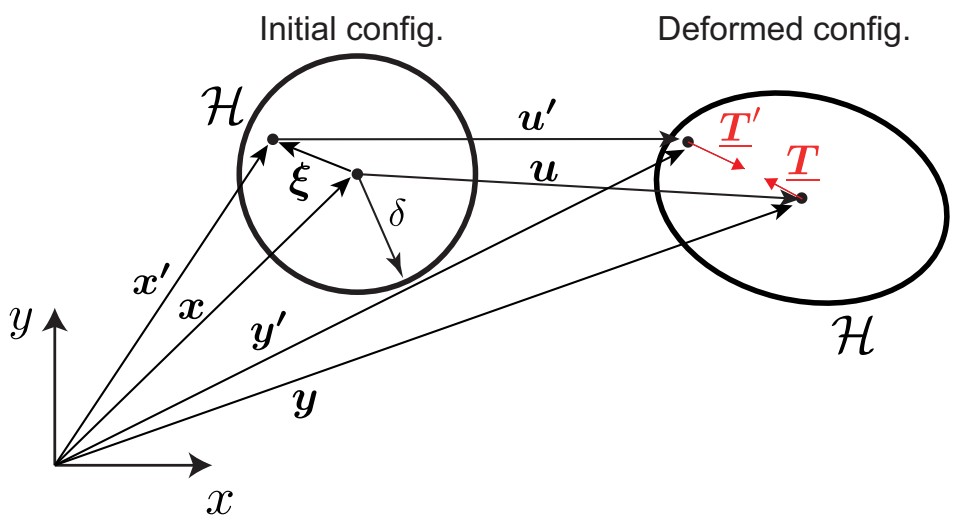

Figure 1: Kinematics of material points in 2D OSB-PD framework.

As illustrated in Fig. 1, the force state of a material point, $\boldsymbol{x}^{\prime}$ within the neighbourhood of a point located at $\boldsymbol{x}$ is different from that of point at $\boldsymbol{x}$, but the force state vectors are aligned with the bond of interacting material points in the deformed configuration. $\mathcal{H}$ stands for the neighbourhood of the point. The horizon size, which defines neighbourhood is denoted by $\delta$. The equation of motion for a material point initially located at $\boldsymbol{x}$ can be written in integral form as:

$$
\rho(\boldsymbol{x}) \ddot{\boldsymbol{u}}(\boldsymbol{x}, t)=\int_{\mathcal{H}}\left[\underline{\boldsymbol{T}}(\boldsymbol{x}, t)\left\langle\boldsymbol{x}^{\prime}-\boldsymbol{x}\right\rangle-\underline{\boldsymbol{T}}\left(\boldsymbol{x}^{\prime}, t\right)\left\langle\boldsymbol{x}-\boldsymbol{x}^{\prime}\right\rangle\right] \mathrm{d} V+\boldsymbol{b}(\boldsymbol{x}, t),
$$

where $\rho(\boldsymbol{x})$ is spatially dependent mass density of the material points, which is important for the modelling of FGMs. $d V$ stands for differential volume for the neighbourhood of point at $\boldsymbol{x}$. $\ddot{\boldsymbol{u}}$ is acceleration vector of the point. The force states are $\underline{\boldsymbol{T}}(\boldsymbol{x}, t)=\underline{\boldsymbol{T}}$ and $\underline{\boldsymbol{T}}\left(\boldsymbol{x}^{\prime}, t\right)=\underline{\boldsymbol{T}}^{\prime}$, which respectively operates on the bond vectors $\left\langle\boldsymbol{x}^{\prime}-\boldsymbol{x}\right\rangle$ and $\left\langle\boldsymbol{x}-\boldsymbol{x}^{\prime}\right\rangle$.

As opposed to bond-based PD theory, force densities of the interacting material points are not necessarily equal to each other, since the force densities of the material points are not only associated with deformation of a bond, but the volume dilatation of interacting material points also affects the force densities. In 2D OSB-PD formulation, the force density vector is defined as [34]:

$$
\underline{\boldsymbol{T}}=\underline{\mathrm{t}} \boldsymbol{n},
$$

where the unit direction vector is $\boldsymbol{n}=\left(\boldsymbol{y}^{\prime}-\boldsymbol{y}\right) /\left|\boldsymbol{y}^{\prime}-\boldsymbol{y}\right|$, in which $\boldsymbol{y}^{\prime}-\boldsymbol{y}$ denotes bond vector in the deformed configuration. The magnitude of the 
force state vector can be expressed for the plane stress condition as [34]:

$$
\underline{t}=\frac{2(2 \nu-1)}{\nu-1}\left(k^{\prime} \theta-\frac{\alpha}{3}\left(\underline{\omega e}^{\mathrm{d}}\right) \bullet|\underline{\boldsymbol{\xi}}|\right) \frac{\underline{\omega}|\underline{\boldsymbol{\xi}}|}{q}+\alpha \underline{\omega e}^{\mathrm{d}},
$$

where $\underline{\omega}$ is the influence function associated with the initial relative position vector as $\underline{\omega}=1-|\boldsymbol{\xi}| / \delta$. The volume dilatation, $\theta$ is given as:

$$
\theta=\frac{2(2 \nu-1)}{\nu-1} \frac{(\underline{\omega}|\underline{\boldsymbol{\xi}}|) \bullet \underline{e}}{q},
$$

Other parameters in Eq. (3) are expressed as:

$$
\begin{gathered}
\alpha=\frac{8 G}{q}, \\
k^{\prime}=K+\frac{G(\nu+1)^{2}}{9(2 \nu-1)^{2}} .
\end{gathered}
$$

In the above equations, dot product of influence function and scalar position state yielding a weighted volume is denoted as $q=(\underline{\omega}|\underline{\xi}|) \bullet|\underline{\xi}|$.

The magnitude of force density vector for the plane strain condition is given by Le et al. [34] as:

$$
\underline{t}=2\left(k^{\prime} \theta-\frac{\alpha}{3} \frac{\omega e^{\mathrm{d}}}{\bullet}|\underline{\boldsymbol{\xi}}|\right) \frac{\underline{\omega}|\underline{\boldsymbol{\xi}}|}{q}+\alpha \underline{\omega e}^{\mathrm{d}},
$$

The volume dilatation for the plane strain condition becomes as:

$$
\theta=2 \frac{(\underline{\omega}|\underline{\xi}|) \bullet \underline{e}}{q} .
$$

The PD parameter, $\alpha$ is same as the plane stress condition, while the other PD parameter, $k^{\prime}$ is expressed as:

$$
k^{\prime}=K+\frac{G}{9} .
$$

The parameters in the expressions given above, $\nu, G$ and $K$ are the Poisson's ratio, shear and bulk modulus, respectively. The scalar extension state is $\underline{e}=\left|\boldsymbol{u}^{\prime}-\boldsymbol{u}\right|$, and its deviatoric part is defined by $\underline{e}^{\mathrm{d}}=\underline{e}-\theta|\underline{\xi}| / 3$. 


\subsection{Modelling of damage}

The peridynamics equation of motion given in Eq. (1) is an integral equation, it is therefore capable of dealing with discontinuities in a solid body. A pre-crack in peridynamics can be defined by eliminating interactions (bonds) between material points across the crack section as shown in Fig. 2. The crack propagation is considered by the critical stretch criterion. For 2D problems, the critical stretch in OSB-PD framework was presented by Madenci and Oterkus [28].

$$
s_{c r}=\sqrt{\frac{G_{c}}{\left(\frac{6}{\pi} G+\frac{16}{9 \pi^{2}}(K-2 G)\right) \delta}}
$$

If the stretch of a bond exceeds the critical stretch value in Eq. (10), it is assumed that the bond is broken.

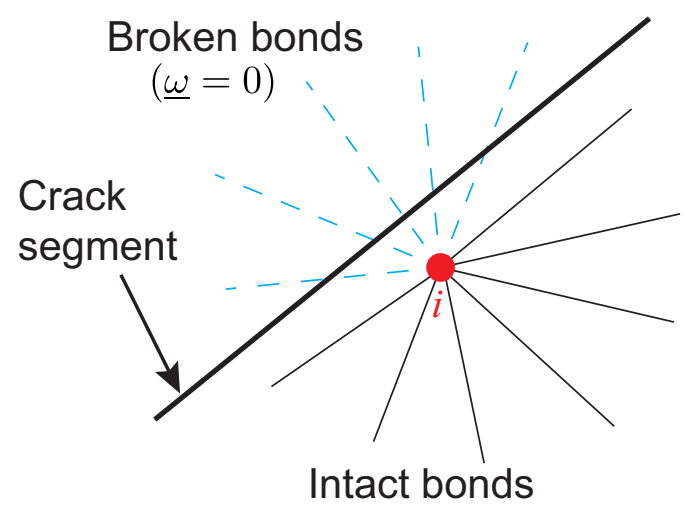

Figure 2: Representation of bond failure in peridynamics.

For a broken bond between the material points, the influence function, $\underline{\omega}$ has to be set as zero. In the critical stretch expression, $G_{c}$ is the critical energy release rate, which can be defined within linear elastic fracture mechanics framework as:

$$
\begin{cases}G_{c}=\frac{K_{\mathrm{IC}}^{2}}{E} & \text { plane stress condition, } \\ G_{c}=\frac{K_{\mathrm{IC}}^{2}(1+\nu)}{E} & \text { plane strain condition, }\end{cases}
$$


where $K_{\mathrm{IC}}$ is the fracture toughness. In case of homogeneous isotropic materials, the fracture toughness and accordingly the critical stretch becomes constant within the solid body. On the other hand, the fracture toughness as well as the critical energy release rate have to be calculated in a point-wise manner for the modelling of FGMs.

For taking into account damage formation and its accumulation at a material point, a scalar step function is utilized to modify the influence function as:

$$
\underline{\omega}=\mu(\boldsymbol{x}, \boldsymbol{\xi}, t)\left(1-\frac{|\boldsymbol{\xi}|}{\delta}\right)
$$

where the step function is adopted as:

$$
\mu(\boldsymbol{x}, \boldsymbol{\xi}, t)= \begin{cases}1 & \text { if } s<s_{c r} \\ 0 & \text { otherwise }\end{cases}
$$

where the stretch is defined by

$$
s=\frac{\left|\boldsymbol{y}^{\prime}-\boldsymbol{y}\right|-\left|\boldsymbol{x}^{\prime}-\boldsymbol{x}\right|}{\left|\boldsymbol{x}^{\prime}-\boldsymbol{x}\right|} .
$$

Local damage parameter is then given as below [13].

$$
\varphi(\boldsymbol{x}, t)=1-\frac{\int_{\mathcal{H}} \mu(\boldsymbol{x}, \boldsymbol{\xi}, t) d V}{\int_{\mathcal{H}} d V} .
$$

The local damage parameter given in Eq. (15) indicates the percentage of the broken bonds belonging to the material point located at $\boldsymbol{x}$. This local damage parameter can be ideally utilized for the assessment process of new crack-surface generation. In this regard, Ha and Bobaru [15] assumed that if the local damage parameter $\varphi(\boldsymbol{x}, t)$ exceeds 0.35 , new crack faces would be generated, which sometimes can be considered as conservative. It is however common practice that the new crack surfaces would be generated, once almost half of the PD bonds on a material point are broken, which corresponds to a critical local damage parameter value of 0.5 [28]. 


\section{Discretization of PD equations}

In the numerical implementation of OSB-PD, the solid body is uniformly discretized in a similar manner with meshfree particle methods. Then, a finite volume is attributed to each material point. The horizon of a material point may not involve the total volume of a neighbouring material point. In this case, the volume correction procedure proposed in [28] is employed. The discretized equation of motion of a material point $(i)$, whose horizon involves the material point $(j)$ with the corrected finite volume $V_{j}^{\prime}$ is given as:

$$
\rho_{(i)} \ddot{\boldsymbol{u}}_{(i)}=\sum_{j}^{N P}\left(\underline{\boldsymbol{T}}_{(i)(j)}-\underline{\boldsymbol{T}}_{(j)(i)}\right) V_{(j)}^{\prime}+\boldsymbol{b}_{(i)},
$$

The force density vectors, $\underline{\boldsymbol{T}}_{(i)(j)}$ and $\underline{\boldsymbol{T}}_{(j)(i)}$ are defined for material points $(i)$ and $(j)$ interacting with each other. Total number of points within the horizon of $(i)$ is denoted by $N P$. The discretized equation of motion can be solved by several explicit time integration schemes. We utilize the central difference scheme, and therefore the acceleration of the material point $(i)$ at time step $t$ is approximated as:

$$
\ddot{\boldsymbol{u}}_{(i)}^{t}=\left(\boldsymbol{u}_{(i)}^{t+1}-2 \boldsymbol{u}_{(i)}^{t}+\boldsymbol{u}_{(i)}^{t-1}\right) /(\Delta t)^{2},
$$

where $\Delta t$ is the time increment size. Introducing Eq. (17) into Eq. (16), the displacement vector for the material point $(i)$ at time step $(t+1)$ is evaluated as:

$$
\boldsymbol{u}_{(i)}^{t+1}=\frac{(\Delta t)^{2}}{\rho_{(i)}}\left[\sum_{j}^{N P}\left(\underline{\boldsymbol{T}}_{(i)(j)}^{t}-\underline{\boldsymbol{T}}_{(j)(i)}^{t}\right) V_{(j)}^{\prime}+\boldsymbol{b}_{(i)}^{t}\right]+2 \boldsymbol{u}_{(i)}^{t}-\boldsymbol{u}_{(i)}^{t-1},
$$

In Eqs. (17) and (18), superscripts represent the time steps. The magnitude of the force state vector for the plane stress condition given by Eq. (3) can be written in discretized form as:

$$
\begin{aligned}
\underline{t}_{(i)(j)}= & \frac{2(2 \nu-1)}{\nu-1}\left(k_{(i)}^{\prime} \theta_{(i)}-\frac{\alpha_{(i)}}{3} \sum_{j}^{N P} \underline{\omega}_{(i)(j)} \underline{e}_{(i)(j)}^{\mathrm{d}}\left|\underline{\boldsymbol{\xi}}_{(i)(j)}\right| V_{(j)}^{\prime}\right) \\
& \times \frac{\underline{\omega}_{(i)(j)}\left|\underline{\boldsymbol{\xi}}_{(i)(j)}\right|}{\sum_{j}^{N P} \underline{\omega}_{(i)(j)}\left|\underline{\boldsymbol{\xi}}_{(i)(j)}\right|\left|\underline{\boldsymbol{\xi}}_{(i)(j)}\right| V_{(j)}^{\prime}}+\alpha_{(i)} \underline{\omega}_{(i)(j)} \underline{e}_{(i)(j)}^{\mathrm{d}},
\end{aligned}
$$


where the dilatation term in discretized form for the plane stress condition can be expressed as:

$$
\theta_{(i)}=\frac{2(2 \nu-1)}{\nu-1} \frac{\sum_{j}^{N P} \underline{\omega}_{(i)(j)}\left|\underline{\boldsymbol{\xi}}_{(i)(j)}\right| \underline{\boldsymbol{e}}_{(i)(j)}^{d} V_{(j)}^{\prime}}{\sum_{j}^{N P} \underline{\omega}_{(i)(j)}\left|\underline{\boldsymbol{\xi}}_{(i)(j)}\right|\left|\underline{\boldsymbol{\xi}}_{(i)(j)}\right| V_{(j)}^{\prime}} .
$$

Similarly for the plane strain condition, the magnitude of force state vector is given in discretized form as:

$$
\begin{aligned}
\underline{t}_{(i)(j)}= & 2\left(k_{(i)}^{\prime} \theta_{(i)}-\frac{\alpha_{(i)}}{3} \sum_{j}^{N P} \underline{\omega}_{(i)(j)} \underline{e}_{(i)(j)}^{\mathrm{d}}\left|\underline{\boldsymbol{\xi}}_{(i)(j)}\right| V_{(j)}^{\prime}\right) \\
& \times \frac{\underline{\omega}_{(i)(j)}\left|\underline{\boldsymbol{\xi}}_{(i)(j)}\right|}{\sum_{j}^{N P} \underline{\omega}_{(i)(j)}\left|\underline{\boldsymbol{\xi}}_{(i)(j)}\right|\left|\underline{\boldsymbol{\xi}}_{(i)(j)}\right| V_{(j)}^{\prime}}+\alpha_{(i)} \underline{\omega}_{(i)(j)} \underline{e}_{(i)(j)}^{\mathrm{d}},
\end{aligned}
$$

Then, the dilatation for the plane strain condition in discretized form can be written as:

$$
\theta_{(i)}=\frac{2 \sum_{j}^{N P} \underline{\omega}_{(i)(j)}\left|\underline{\boldsymbol{\xi}}_{(i)(j)}\right| \underline{\boldsymbol{e}}_{(i)(j)}^{d} V_{(j)}^{\prime}}{\sum_{j}^{N P} \underline{\omega}_{(i)(j)}\left|\underline{\boldsymbol{\xi}}_{(i)(j)}\right|\left|\underline{\boldsymbol{\xi}}_{(i)(j)}\right| V_{(j)}^{\prime}} .
$$

In order to consider fracture and crack propagation in OSB-PD framework, if the stretch value between material points $(i)$ and $(j)$ exceeds the critical stretch given in Eq. (10), the bond breakage is taken into account by setting $\underline{\omega}_{(i)(j)}=0$ in the above equations.

The local damage parameter for a material point $(i)$ can be expressed in discretized form at time $t$ as:

$$
\varphi_{(i)}^{t}=1-\frac{\sum_{j}^{N P} \mu_{(i)(j)}^{t} V_{(j)}^{\prime}}{\sum_{j}^{N P} V_{(j)}^{\prime}} .
$$




\section{Modelling of FGMs}

FGMs are special kind of composite materials of which the material properties change smoothly over the thickness or width direction. Such material models are promising for avoiding delamination and stress discontinuities as compared to conventional laminated composites. Simultaneously applied thermal and mechanical loads can also be sustained by FGMs owing to its different material properties of each faces.

Considering the change of material properties over width/thickness direction, some assumptions are adopted in the present study to determine force states and fracture parameters. When evaluating the magnitude of force state vectors in Eqs. (19) and (21), the dilatation, $\theta$ is determined for the point of interest $(i)$. On the other hand, the peridynamic parameters, $\alpha$ and $k^{\prime}$ are function of the material properties and the averaging technique given by Eq. (24) is employed for calculating the material properties for the peridynamic parameters, $\alpha$ and $k^{\prime}$.

$$
P_{\text {bond }}=\frac{P_{(i)}+P_{(j)}}{2},
$$

where $P_{\text {bond }}$ stands for averaged value of material parameter for the bond between the material points $(i)$ and $(j) . P_{(i)}$ and $P_{(j)}$ respectively represent the material parameters at the points $(i)$ and $(j)$. The averaging procedure is schematically illustrated in Fig. 3

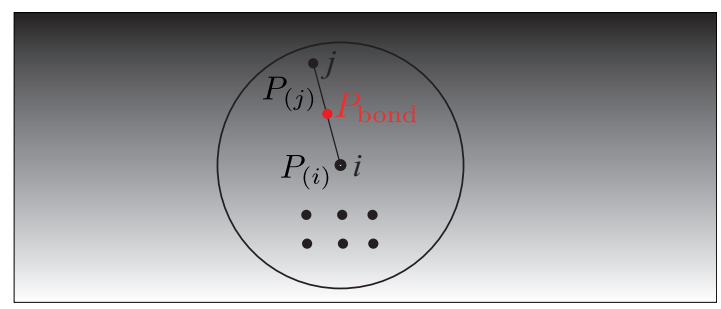

Figure 3: Averaging of material parameters in FGMs.

In accordance with the procedure described above, the parameter $\alpha$ for a bond in the modelling of FGMs is approximated as:

$$
\alpha=\frac{8\left(G_{(i)}+G_{(j)}\right)}{2 q},
$$


where $G_{(i)}$ and $G_{(j)}$ are the shear modulus of the material points at each side of the PD bond. In Eq. (25), $q$ is independent of the material properties, it only depends on the discretization and weight function. Apart from this, the parameter $k^{\prime}$ is a function of the material properties, and for approximating this parameter, the averaged values of Poisson's ratio, shear and bulk moduli are introduced.

To obtain critical stretch for the bond, the critical energy release rate, $G_{c}$ in Eq. (10) is calculated by averaging of critical energy release rates at the material points $(i)$ and $(j)$. In addition, shear and bulk moduli in Eq. (10) are also averaged values of those at interacting material points. For minimizing the error due to the averaging procedure, the distance between the material points and the horizon size must be selected small enough.

\section{Numerical studies}

In this section, we aim to show the performance of our computational model on capturing the fracture/damage of FGMs as well as homogeneous plates. First, we revisit a well known benchmark crack branching problem. Then, we perform various dynamic analysis to validate current approach for modelling dynamic wave propagation in FGM structures. Finally, the dynamic fracture of FGMs is studied. Both crack branching and simulation of triple point bending (TPB) experiments in FGMs are addressed.

The displacement boundary conditions are not necessary to enforce in the PD framework since the rigid body motion can be avoided by the equation of motion. If any specific displacement boundary condition is not prescribed in the problems, then the problem is handled under the given loading conditions without any displacement constraints.

The general solution procedure followed in the present work is schematically illustrated in Fig. 4, which can be applied to the all numerical examples in the present work. In case of the homogeneous material, the material properties as well as the critical stretch value would be the same for all bonds. As for the wave propagation problem in FGMs, "Assessment of crack propagation" step in the red frame is skipped. In the evaluation of crack propagation in FGMs, every step given in Fig. 4 is followed. 


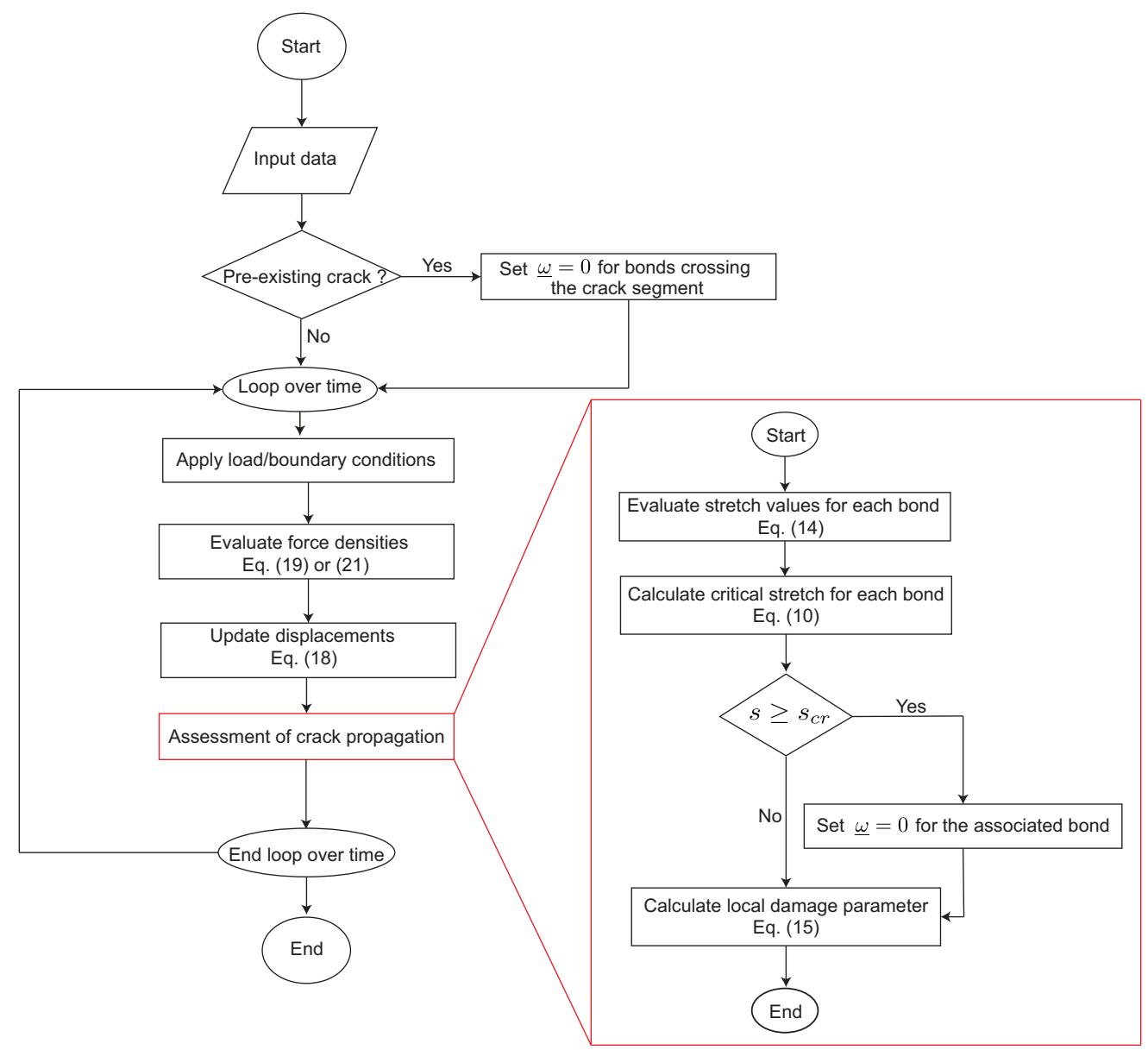

Figure 4: General solution procedure implemented through the numerical examples. 


\subsection{Benchmark study for crack branching in homogeneous materials}

In this example, crack branching in a homogeneous plate is studied. This is a well-known benchmark problem, which was experimentally studied by Bowden et al. [35] and then the problem was examined by the bond-based PD model in Refs. $[15,16]$. A rectangular plate with a pre-crack as shown in Fig. 5(a) is considered.

(a)

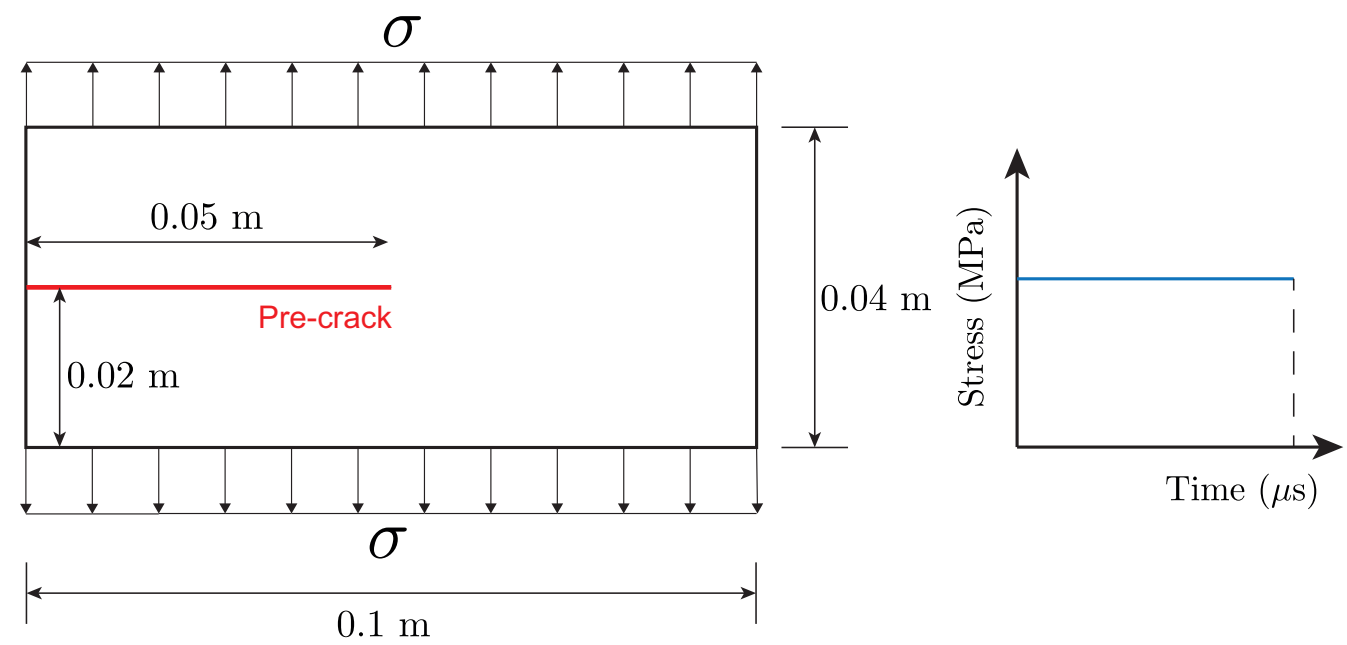

Figure 5: Modelling of homogeneous plate for crack branching: (a) geometry, (b) loading profile.

The material properties as well as the simulation procedure are chosen as same as those given by Ref. [15] with the aim of comparing crack path and the average crack speed. Soda-lime glass material is adopted for simulating crack branching by using OSB-PD. The material properties are considered as follow. The mass density is $\rho=2440 \mathrm{~kg} / \mathrm{m}^{3}$, elastic modulus, Poisson's ratio and critical energy release rate are taken as $E=72 \mathrm{GPa}, \nu=0.22$ and $G_{c}=135 \mathrm{~J} / \mathrm{m}^{2}$, respectively [15].

The rectangular plate given in Fig. 5(a) is uniformly discretized by material points as similar to meshfree particle methods. Defining the horizon size as $\delta=4 \times \Delta x$; the medium $(\delta=1 \mathrm{~mm})$ and large horizon $(\delta=2$ $\mathrm{mm})$ sizes are considered. $\Delta x$ represents the distance between the material points. Loading condition and time increment size are assumed as same as those of Ref. [15], in which the time step size is $\Delta t=25 \mathrm{~ns}$ and the total 
simulation time is considered as $40 \mu \mathrm{s}$. A symmetric tensile load, $\sigma=14$ $\mathrm{MPa}$ is suddenly applied to upper and lower edges of the plate until the end of simulation, see Fig. 5(b). Plane stress condition is adopted in the simulations.

Crack tip location is recorded for $2 \mu$ s uniform time intervals and the average crack speed is evaluated. Ha and Bobaru [15] assumed that the crack propagation occurs when the local damage parameter of a material point exceeds 0.35, and this assumption is utilized in the present numerical example for comparison purposes. The crack speeds for medium and large horizons obtained by the present OSB-PD modelling are compared with those of Ref. [15] in Fig. 6. The reference values are extracted from graphical data given by Ref. [15]. In the given figure, the solid red line is the maximum experimental crack speed, $1580 \mathrm{~m} / \mathrm{s}$ given by Bowden et al. [35].

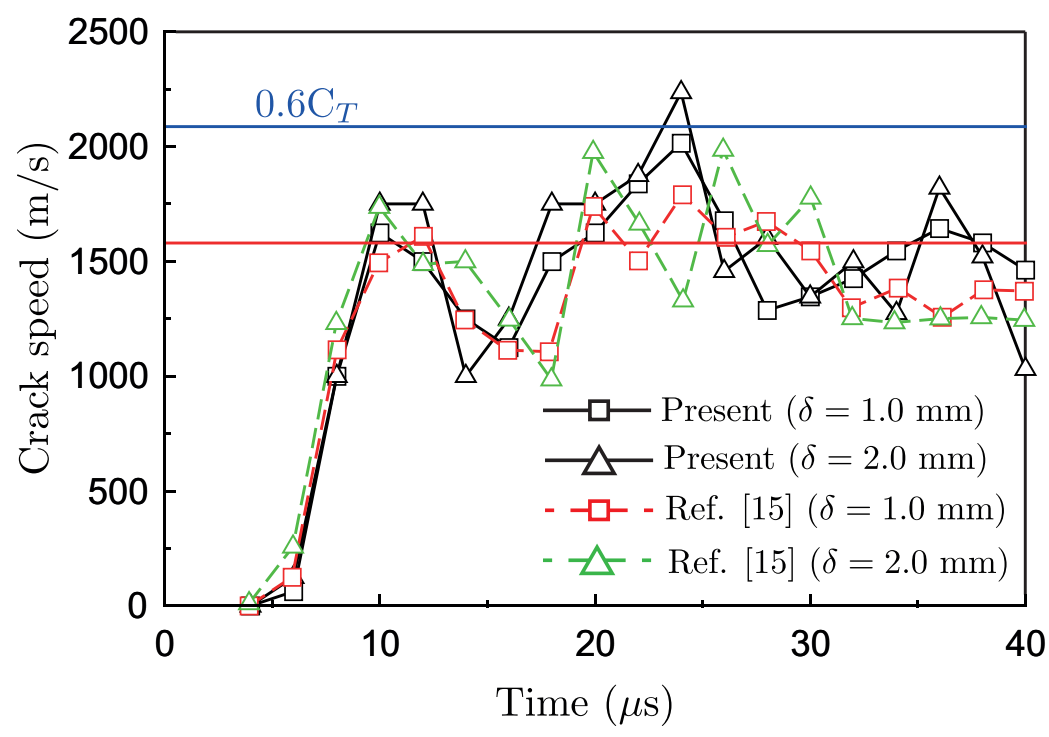

Figure 6: Comparison of crack speeds obtained by present approach and reference works.

The average crack speeds evaluated by the present OSB-PD modelling show relatively good agreement with the reference numerical results and the experimental value. The onset of crack propagation for the large horizon size in Ref. [15] is earlier than the present models. The possible reason is the different critical stretch values calculated by Eq. (10) and the adopted one 
in Ref. [15]. Moreover, the critical stretch values also have consequences on the measured crack speeds.

For verifying our computational model in an analytical manner, approximate crack branching speed is studied. The crack branching speed is approximately given as, $\dot{a}_{\mathrm{br}} \approx 0.6 C_{T}[36]$. Here, $C_{T}$ is the transverse wave speed in solids, which can be written as [36]:

$$
C_{T}=\sqrt{\frac{G}{\rho}},
$$

where $G$ and $\rho$ denote shear modulus and mass density, respectively. When the material properties are introduced into the above equation, the crack branching speed is approximately evaluated as $2086 \mathrm{~m} / \mathrm{s}$, which is shown by solid blue line in Fig. 6. In this problem, the crack branching takes place beyond $20 \mu$ s as shown in Fig. 7, and the increase of the crack speed beyond $20 \mu$ s can be observed in Fig. 6. The maximum crack speeds around the branching point are 2015 and $2236 \mathrm{~m} / \mathrm{s}$ for medium and large horizon sizes, respectively. These values are consistent with the given approximation, $\dot{a}_{\mathrm{br}} \approx 0.6 C_{T}$.

Estimated crack paths obtained for the medium and large horizon sizes are given in Fig. 7. The given figure apparently shows that the estimated crack paths are in good agreement with those in Ref. [15]. The onset of crack branching for both horizon sizes is observed beyond $t=20 \mu \mathrm{s}$. This value is also consistent with the work of Ha and Bobaru [15]. 
(a)

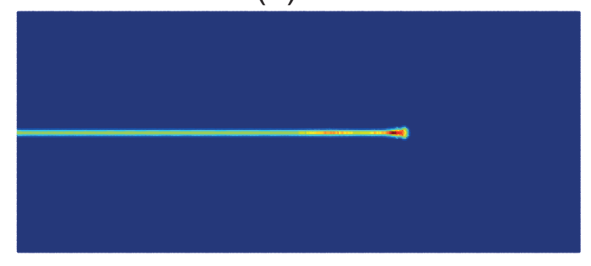

$t=20 \mu \mathrm{s}$

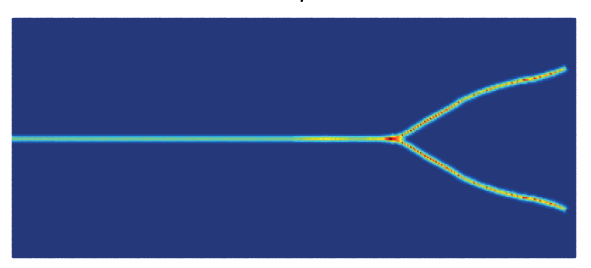

$t=40 \mu \mathrm{s}$ (b)

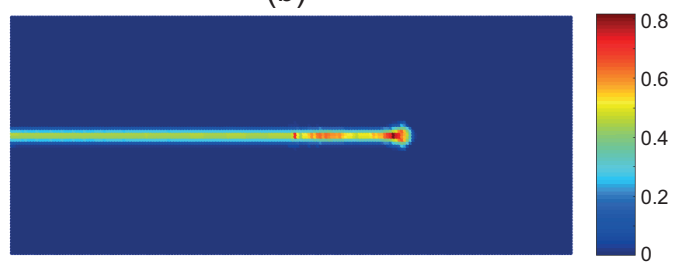

$t=20 \mu \mathrm{s}$

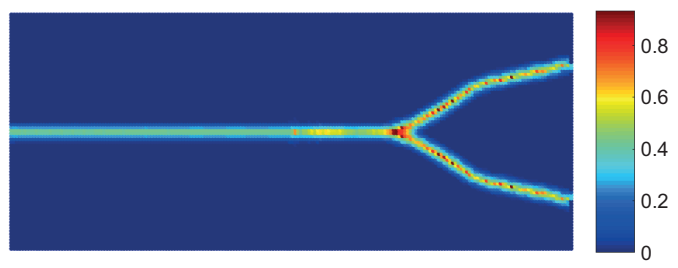

$t=40 \mu \mathrm{s}$

Figure 7: Estimated crack paths for the homogeneous plate: (a) medium horizon size $(\delta=1 \mathrm{~mm})$, (b) large horizon size $(\delta=2 \mathrm{~mm})$.

\subsection{Wave propagation in FGMs under impact loading}

So far, the brittle crack propagation and the crack branching in homogeneous materials were examined, the reliability and accuracy of our modelling have been verified.

The displacement wave propagation of FGMs is studied in this section before proceeding to fracture problems in FGMs. Displacement wave propagation is a crucial natural phenomenon to investigate the crack propagation, since these waves may trigger the crack propagation.

Hedayatrasa et al. [3] carried out dynamic analyses for FGMs using the spectral element method. The specimens for the wave propagation problem are adopted from Ref. [3]. The same wave propagation problems were studied by Cheng et al. [23] using the bond-based PD modelling. The representative model and loading condition are illustrated in Fig. 8. Similar to Ref. [3], the present OSB-PD simulations of the wave propagation problems are performed based on the assumption of plane strain condition. 

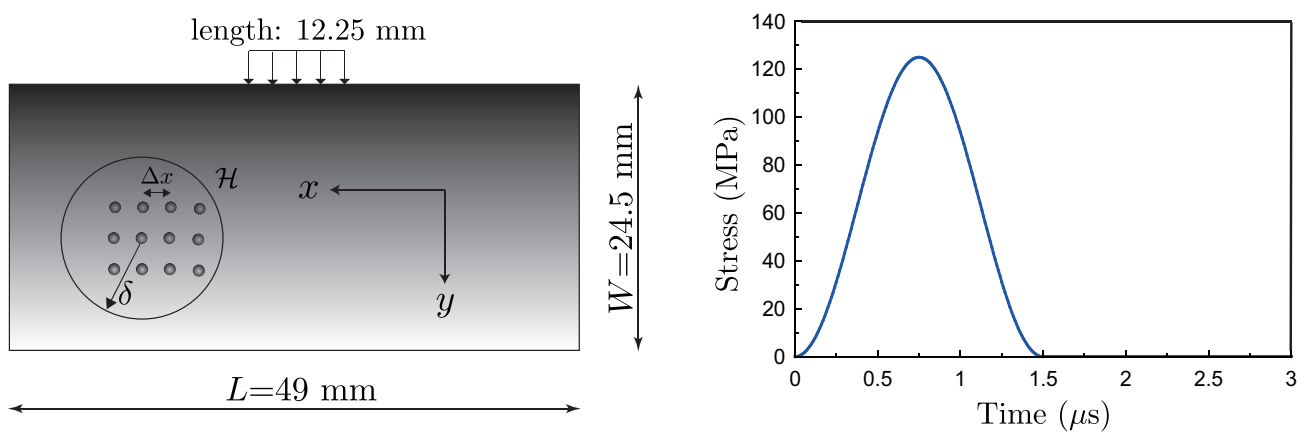

Figure 8: Modelling and loading conditions for wave propagation in FGMs.

The material properties are as same as Ref. [3], and listed in Table 1. The top surface is the ceramic rich zone, i.e., stiff side of the specimen, while the bottom surface is metallic side of the specimen. The composition of the ingredients is controlled by power law distribution as given by Ref. [3].

$$
\begin{gathered}
\phi(y)=\phi_{0}\left(\frac{y}{W}\right)^{d}, \quad 0 \leq y \leq W, \\
P_{\text {eff }}=P_{1} \phi(y)+P_{2}(1-\phi(y)),
\end{gathered}
$$

where $P_{\text {eff }}$ stands for the point-wise effective material property. $P_{1}$ and $P_{2}$ respectively denote the material property for phase 1 (ceramic) and phase 2 (metal). Three different composition ratios are taken into account in the wave propagation problems. Case 1 represents the uniform composition with the parameters $\phi_{0}=0.36$ and $d=0$. The parameter, $\phi_{0}=0.6$ is utilized for Cases 2 and 3, while $d=0.25$ and 4 are considered for Cases 2 and 3, respectively.

Table 1: Material properties of FGMs for wave propagation [3].

\begin{tabular}{ccc}
\hline Parameter & Phase-1 (top) & Phase-2 (bottom) \\
\cline { 2 - 3 } & Ceramic (Alumina) & Metal (Aluminum) \\
\hline$E(\mathrm{GPa})$ & 420 & 70 \\
$\nu$ & 0.17 & 0.3 \\
$\rho\left(\mathrm{kg} / \mathrm{m}^{3}\right)$ & 3200 & 2800 \\
\hline
\end{tabular}


The loading profile plotted in Fig. 8 is obtained by the following expression $[3]$.

$$
\sigma(t)=\sigma_{0} \sin ^{2}\left(\pi \frac{t-2 t_{r}}{2 t_{r}}\right),
$$

where $\sigma_{0}=125 \mathrm{MPa}$ and the duration of the half load cycle is $t_{r}=0.75 \mu \mathrm{s}$. The total simulation time is $t_{\text {tot }}=3.0 \mu \mathrm{s}$. The FGM specimen is discretized by $240 \times 120$ points with uniform discretization size, $\Delta x$. The horizon size is assumed as $\delta=3.015 \times \Delta x$ and the time increment is taken as $\Delta t=0.01 \mu \mathrm{s}$. The displacement contours after $3.0 \mu$ s are given in Fig. 9.

(a)
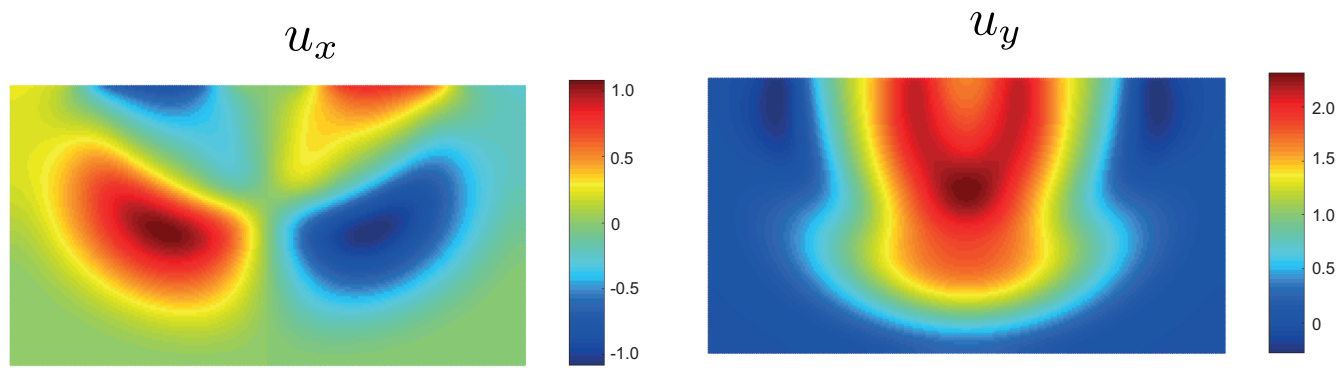

(b)
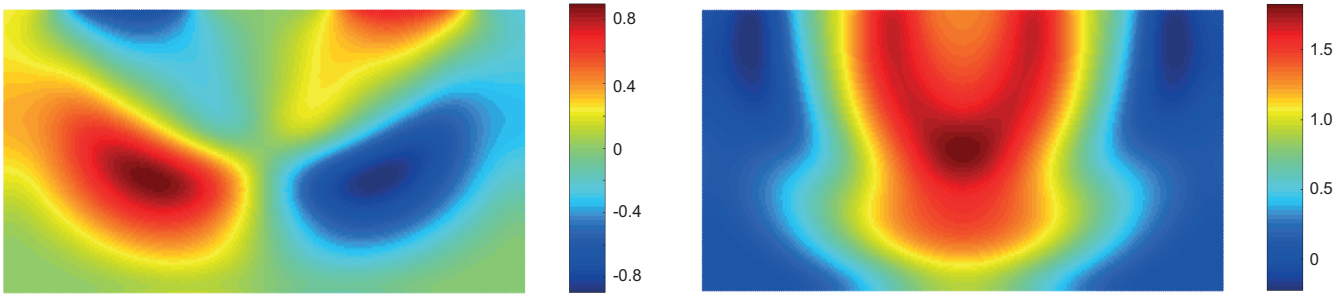

(c)
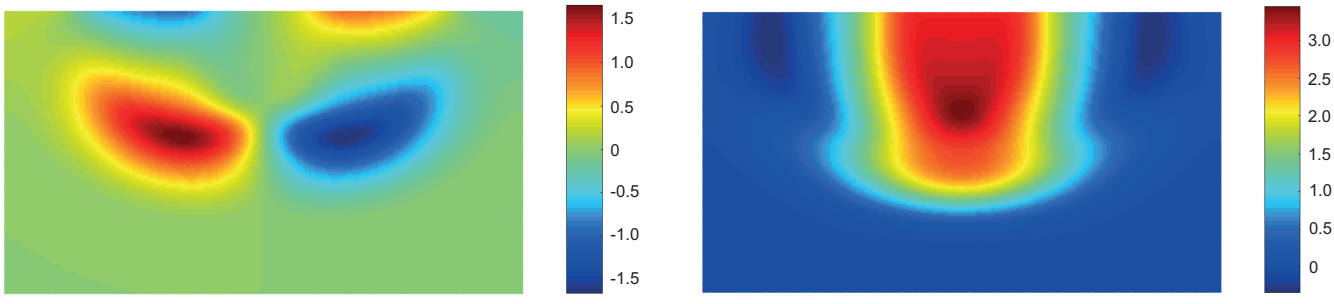

Figure 9: Displacement wave contours ( $\mu \mathrm{m}$ ) in FGMs: (a) Case 1, (b) Case 2, (c) Case 3.

Fig. 9(a) shows the displacement contours for the uniformly reinforced FGMs, Case 1. The wave top of the vertical displacement cannot reach the 
bottom surface, and this behaviour was also observed in Ref. [3]. As for the Case 2, volume fraction of the ceramic reinforcement increases dramatically at the lower side of the specimen, and the wave top of vertical displacement reaches the bottom surface as indicated in Fig. 9(b); however the maximum magnitude of the vertical displacement is lower compared to Case 1. For the last composition ratio, Case 3, top of vertical displacement wave cannot reach the bottom surface, however the maximum magnitude of vertical displacement is the largest compared to other cases. For all composition ratios considered, the obtained displacement contours agree well with those in Ref. [3], thus demonstrating the high predictive capabilities of present OSB-PD computational model for the dynamic impact analysis of FGM materials.

\subsection{Crack branching in FGMs}

For the dynamic fracture in FGMs, the crack branching problem depicted in Fig. 10 is examined first. This problem was studied by Ref. [24] through employing the bond-based PD.

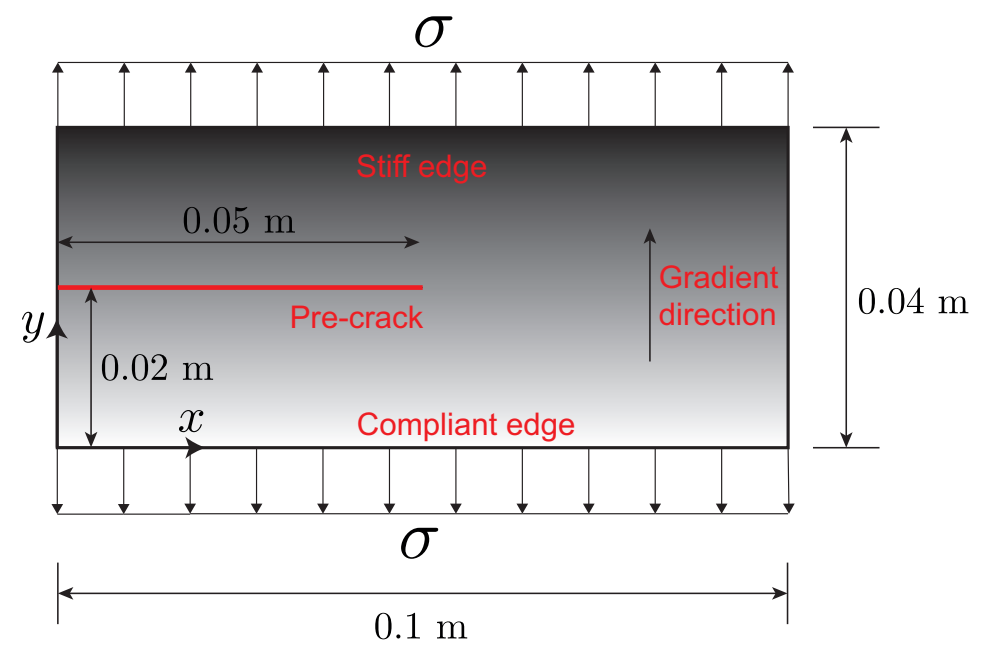

Figure 10: Modelling and loading conditions for crack branching problem in FGMs.

As it is given in Fig. 10, the bottom is the compliant side while the top surface is the stiff side. The material properties are varied linearly as assumed in Ref. [24], and described as:

$$
K_{\mathrm{IC}}(y)=2.1+\frac{3.6-2.1}{40} y
$$




$$
\begin{aligned}
& E(y)=3.8+\frac{11.1-3.8}{40} y, \\
& \rho(y)=918+\frac{1812-918}{40} y .
\end{aligned}
$$

In the above equations, $y$ is varied between 0 and $40 \mathrm{~mm}$. The units for fracture toughness, elastic modulus and mass density are MPa.m ${ }^{1 / 2}$, GPa and $\mathrm{kg} / \mathrm{m}^{3}$, respectively. Cheng et al. [24] considered the plane stress condition for this problem. Accordingly, the same assumption is adopted in the present work. Poisson's ratio is utilized as constant, $\nu=1 / 3$. In order to determine the critical stretch in Eq. (10), the spatially dependent critical energy release rate is obtained under plane stress condition by

$$
G_{c}(y)=\frac{K_{\mathrm{IC}}^{2}(y)}{E(y)} .
$$

The critical energy release rates are evaluated by Eq. (33) for the material points located at each side of the bond, those values are then averaged by the procedure given in Eq. (24). The averaged value is introduced into Eq. (10) to obtain the critical stretch value of the bond.

Symmetric tensile pressure load of magnitude $\sigma=15 \mathrm{MPa}$ is simultaneously applied on both top and bottom edges of the specimen. The loading procedure is same as the homogeneous plate case as given in Fig. 5(b). Stable time step size assumed by Cheng et al. [24] is $\Delta t=50 \mathrm{~ns}$; however in the present work, two different time step sizes, $\Delta t=20,50 \mathrm{~ns}$, are considered with two different horizon sizes, $\delta=4 \times \Delta x=1.6,2.0 \mathrm{~mm}$. Total simulation time is assumed as $60 \mu \mathrm{s}$. Both crack paths and the average crack speeds are compared with each other as well as the reference work.

Fig. 11 indicates the average crack speeds for the FGM specimens. To calculate the average crack speed, it is assumed that the crack propagation occurs when the local damage parameter, $\varphi(\boldsymbol{x}, t)$ exceeds 0.5 [28]. Then, the coordinates of the material points whose local damage parameter exceeds the given value are recorded as the crack tip location. The crack tip location is measured for uniform time intervals, $4 \mu \mathrm{s}$. Considering the crack tip locations obtained by the mentioned procedure, the averaged crack speeds are evaluated. The crack speed is calculated for the lower tip of the crack after crack branching takes place.

In all cases, the crack propagation starts in a close range, which is around 14-15 $\mu \mathrm{s}$. The onset of crack propagation is decided by checking the local 
(a)

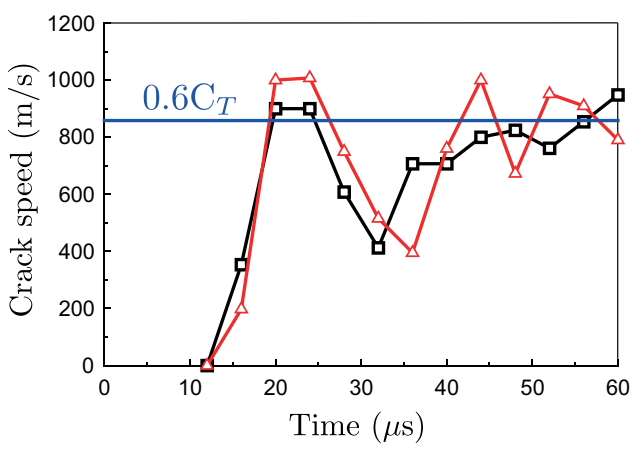

(b)

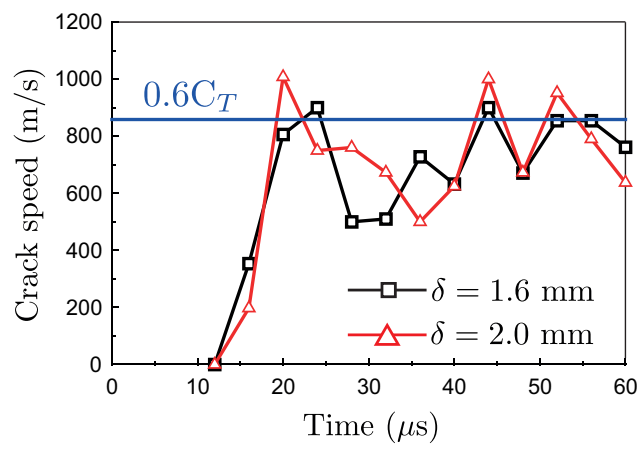

Figure 11: The average crack speeds for crack branching in FGMs: (a) $\Delta t=20 \mathrm{~ns}$, (a) $\Delta t=50$ ns.

damage parameters of the material points. It is assumed that at the first instance when local damage parameter of a material point exceeds 0.5 , the onset of crack propagation is achieved. Accordingly, the onsets of crack propagation are obtained in $\delta=1.6 \mathrm{~mm}$ division case as $15.28,15.40 \mu \mathrm{s}$ for $\Delta t=20,50 \mathrm{~ns}$, respectively; while $14.18,14.50 \mu$ s are the beginning of crack propagation in case of $\delta=2.0 \mathrm{~mm}$ for $\Delta t=20,50 \mathrm{~ns}$, respectively.

General trend of the crack speeds are in good agreement for all division and time step size cases. The crack speed just after the crack propagation systematically increases until $20 \mu \mathrm{s}$, which is associated with the elastic strain energy stored in the specimens prior to crack propagation.

Initially, the crack propagates through a straight path, then the crack tip inclines towards the compliant side. This behaviour was not observed in the homogeneous case in which the crack path is symmetric with respect to horizontal line along the pre-crack. Unlike the homogeneous case, the main reason for the non-symmetric crack path is the variation of the material properties as well as the critical stretch from bottom to top edge. In the bottom side, the fracture toughness and the critical stretch values are lower compared to the upper side of the specimen; the crack then propagates towards the bottom side.

The crack branching takes place around 44-45 $\mu$ s for all cases. Once crack branching takes place, the crack speed increases slightly owing to both vertical and horizontal displacements of the crack tip. After the occurrence of crack branching, it can be easily observed that the length of the lower branch 
is slightly higher than that of the upper branch, which is also associated with the variation of the material properties.

The branching crack speed discussed in the previous section is valid for homogeneous materials. As for the FGMs, assuming the variation of material properties within neighbourhood of the pre-crack is smooth, the approximate crack branching speed can be obtained for $E=7.45 \mathrm{GPa}$, and $\rho=1365$ $\mathrm{kg} / \mathrm{m}^{3}$ corresponding the material property values for $y=20 \mathrm{~mm}$ in Eqs. (31) and (32). The approximate crack branching speed is then evaluated as $858 \mathrm{~m} / \mathrm{s}$, which is also shown in Fig. 11 by solid blue line. The crack branching speed can be regarded as the limiting crack speed. In the given figure, the average crack speeds for the large discretization size exceeds the branching speed. This is because, the crack tip jumps from one material point to another one when the enough number of bonds are broken; if the distance between the material points is large, the average crack speeds may exceed the theoretical value. On the other hand, the maximum crack speeds and the crack speeds at the instance of branching are consistent with the theoretical value in case of smaller discretization size. These obtained values suggest that the fine particle discretization reveals more consistent estimations for the branching and limiting crack speeds. When $\Delta t=50 \mathrm{~ns}$, the crack speed for $\delta=2.0 \mathrm{~mm}$ exceeds the limiting value for three times, then the damage patterns for those instances are given in Fig. 12 .

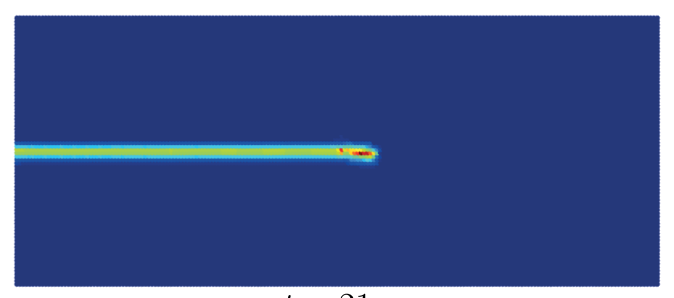

$t=21 \mu \mathrm{s}$

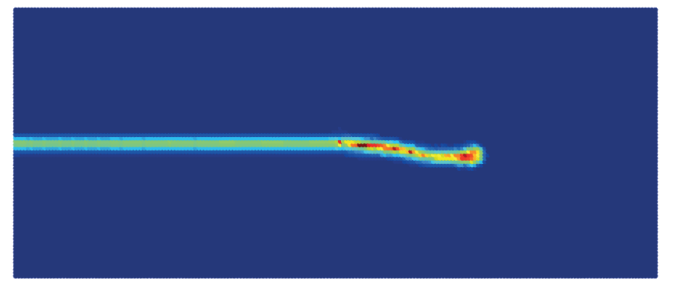

$$
t=45 \mu \mathrm{s}
$$

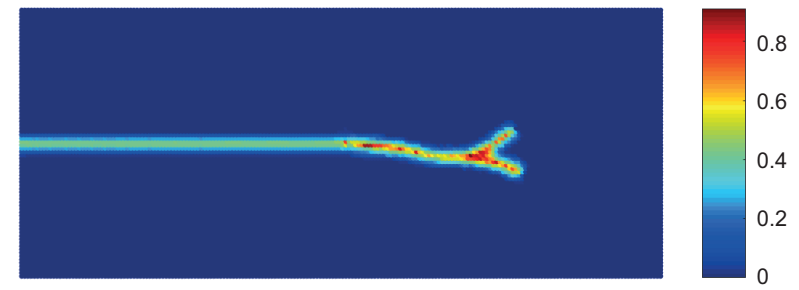

$t=52 \mu \mathrm{s}$

Figure 12: Damage patterns for $\delta=2.0 \mathrm{~mm}$ with $\Delta t=50 \mathrm{~ns}$ time step size. 
The main reason for larger crack speeds is the large distance between the material points. At the instance of $t=21 \mu \mathrm{s}$, the crack tip inclines towards the downward direction. At this moment, the crack speed exceeds the limiting value. According to the crack speed, the local crack branching occurs at the moment. However, the direction of crack propagation is along the main crack. After the crack is propagated towards the compliant side, the crack branching can now occurs around $t=45 \mu \mathrm{s}$. This is because, the fracture toughness of the crack tip at the moment is lower compared that of the pre-crack tip. Beyond the crack branching, the average crack speed is measured for the lower crack tip. At the instance of $t=52 \mu \mathrm{s}$, the double crack tip is observed. Beyond this point, the lower crack tip broadens, which visually becomes more clear in the damage patterns at the end of simulation in Fig. 13(b) for $\Delta t=50 \mathrm{~ns}$.

(a)

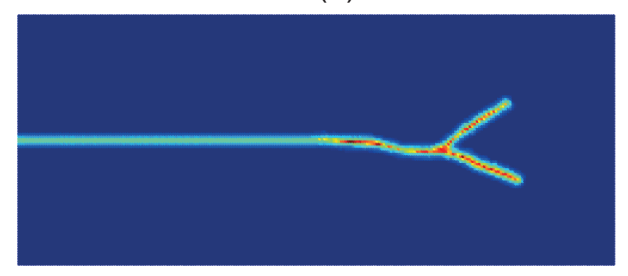

$\Delta t=20 \mathrm{~ns}$

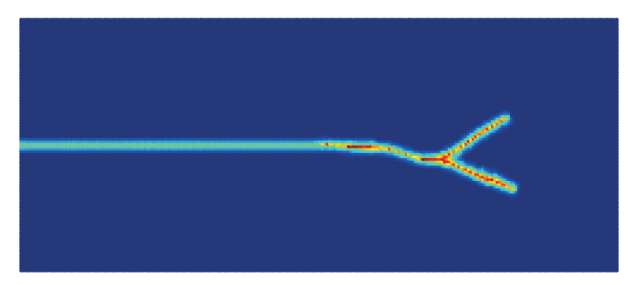

$\Delta t=50 \mathrm{~ns}$ (b)

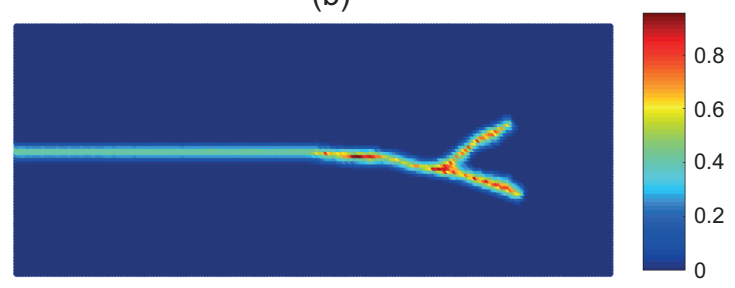

$\Delta t=20 \mathrm{~ns}$

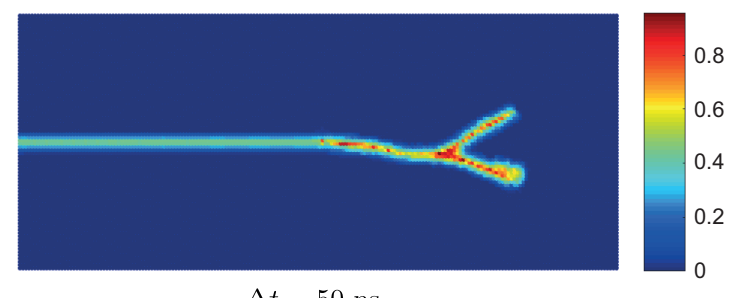

$\Delta t=50 \mathrm{~ns}$

Figure 13: The crack path estimations in FGMs at the end of simulation: (a) $\delta=1.6 \mathrm{~mm}$, (b) $\delta=2.0 \mathrm{~mm}$.

The crack paths at the end of loading $(t=60 \mu \mathrm{s})$ are given in Fig. 13. The crack patterns are consistent with each other, but for the smaller division size, the crack lines become more distinct. Furthermore, the crack patterns obtained by the present model are in good agreement with those presented in Ref. [24]. 


\subsection{Simulation of triple-point bending (TPB) experiments in FGMs}

We simulate TPB experiments by OSB-PD formulation in this section. This problem was experimentally studied by Kirugulige and Tippur [6], the FEM simulations were then conducted by the same authors employing the cohesive zone model [7]. The TPB model is schematically illustrated in Fig. 14. In the given figure, the distributed pressure load on the top surface is applied over $6 \mathrm{~mm}$ width, while the symmetric bottom pressures are applied over $3 \mathrm{~mm}$ width. The crack is located on the lower edge of the specimen.

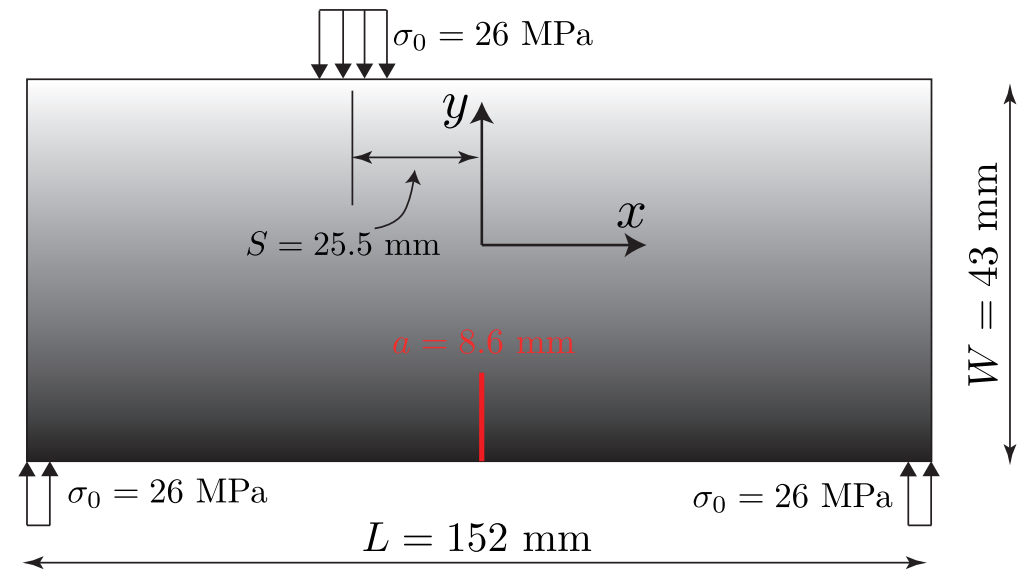

Figure 14: TPB model for FGMs.

Since whole details of the loading procedure and loading time were not provided in Ref. [6], Cheng et al. [23] made some assumptions on the loading procedure. The load magnitude and loading procedure are adopted from Ref. [23] for the comparison of the numerical results in addition to the experiments [6]. In Ref. [23], the pressure load was applied as "rump-up-down" profile; however in the present work, we take into account both rump-up-down and constant loading conditions as indicated in Fig. 15 so as to examine the crack propagation precisely .

In case of rump-up-down loading condition, rump-up and rump-down durations are same, $30 \mu \mathrm{s}$. Four different total loading durations are considered, $t_{\text {load }}=100,150,180,200 \mu$ s irrespective of the loading profiles. Total simulation time is utilized as $300 \mu \mathrm{s}$ in order to capture full failure of the specimens for all loading and division cases. The horizon size is utilized as 

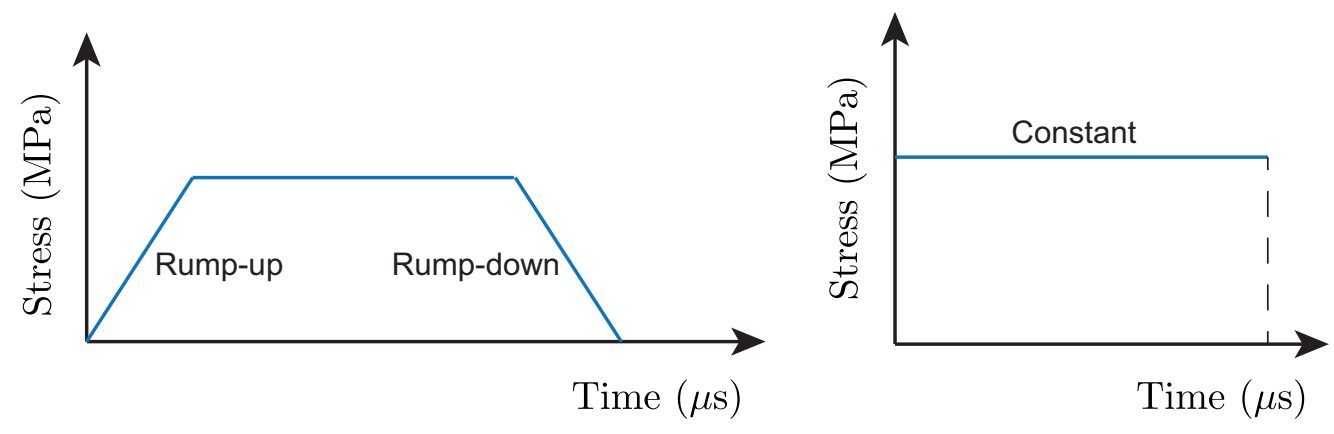

Figure 15: Loading profiles for TPB simulations.

$\delta=4 \times \Delta x=2.0 \mathrm{~mm}$. The crack is considered to be located on either stiff side or compliant side of the specimen.

\subsubsection{Crack on stiff side}

In this section, the lower edge of the specimen is assumed as the stiff side. The material properties are varied linearly as follows [23].

$$
\begin{gathered}
K_{\mathrm{IC}}(y)=2.2-\frac{2.2-1.4}{43} y \\
E(y)=10-\frac{10-4}{43} y \\
\rho(y)=1750-\frac{1750-1175}{43} y
\end{gathered}
$$

where $y$ is varied between 0 and $43 \mathrm{~mm}$. The units for fracture toughness, elastic modulus and mass density are $\mathrm{MPa} \cdot \mathrm{m}^{1 / 2}, \mathrm{GPa}$ and $\mathrm{kg} / \mathrm{m}^{3}$, respectively. Poisson's ratio is assumed to be constant as $\nu=0.34[6,7]$. Critical time step size is presumed as $\Delta t=20 \mathrm{~ns}$ in consistent with Ref. [23]. Critical energy release rate is determined by Eq. (33), and the evaluation of bond properties is carried out in the same manner with the crack branching problems as given in the previous section.

The crack path estimations are given in Fig. 16. The evaluated crack patterns are in relatively good agreement with those of Ref. [23]. It should be noted that Cheng et al. [23] employed the bond-based PD formulation. Moreover, the critical stretch values obtained within OSB-PD framework and the bond-based PD framework would be different. Fig. 16 clearly shows that 


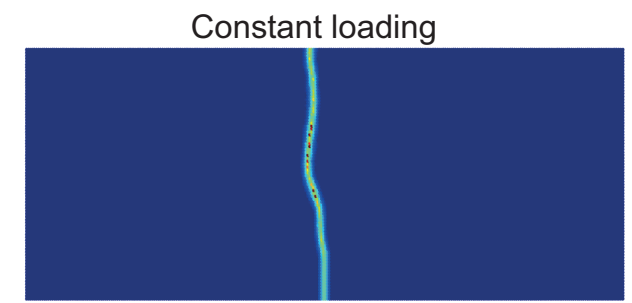

$t_{\text {load }}=100 \mu \mathrm{s}$

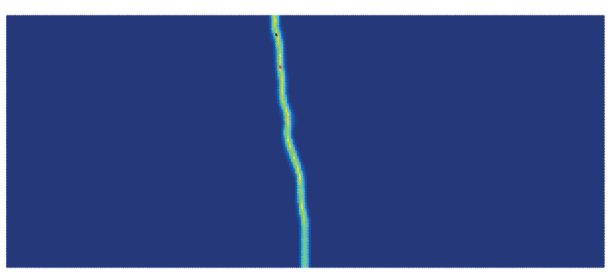

$t_{\text {load }}=150 \mu \mathrm{s}$

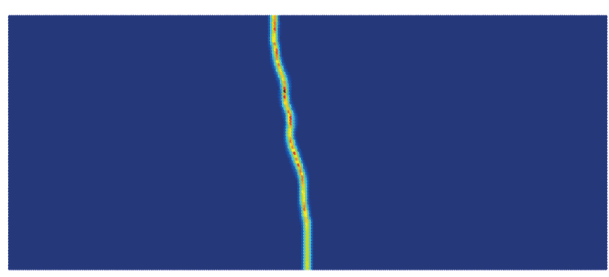

$t_{\text {load }}=180 \mu \mathrm{s}$

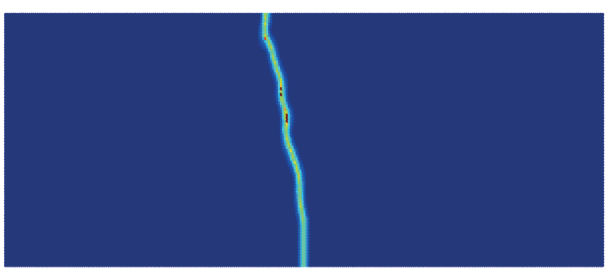

$t_{\text {load }}=200 \mu \mathrm{s}$
Rump-up-down loading
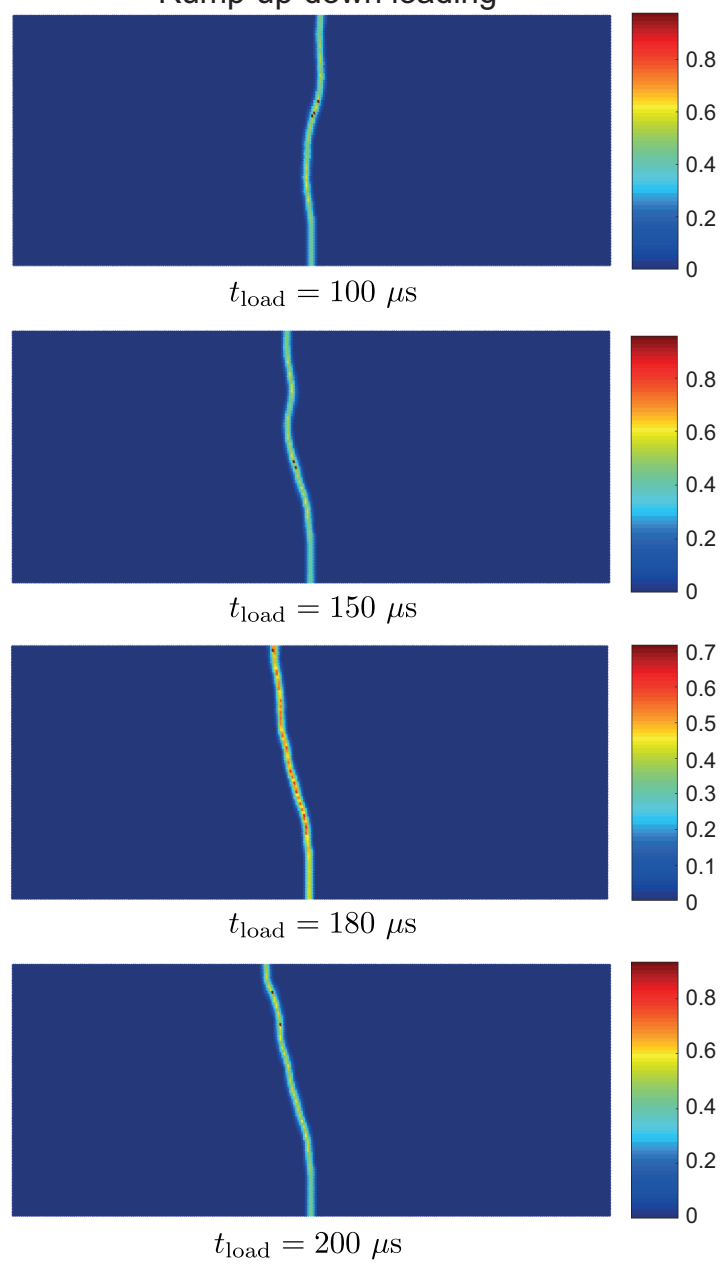

Figure 16: Crack patterns of FGMs in which the crack is located on the stiff side for different loading profiles and durations. 
the crack patterns are significantly affected by both loading duration and the loading profile. It can be inferred from Fig. 16 that more consistent crack patterns with the experiments [6] and the numerical studies [23] are obtained for $t_{\text {load }}=180,200 \mu$ s total loading durations.

Dynamic fracture of the present specimens were also simulated by Doan et al. [10] employing hybrid phase field model. However, Ref. [10] has taken into account either constant or linear velocity conditions over the partial length of upper surface as the loading procedure. We digitized crack path figures from the experiments and phase field model results presented by Ref. [10]. Then, the digitized values are compared with the present crack path estimations in Fig. 17.

(a)

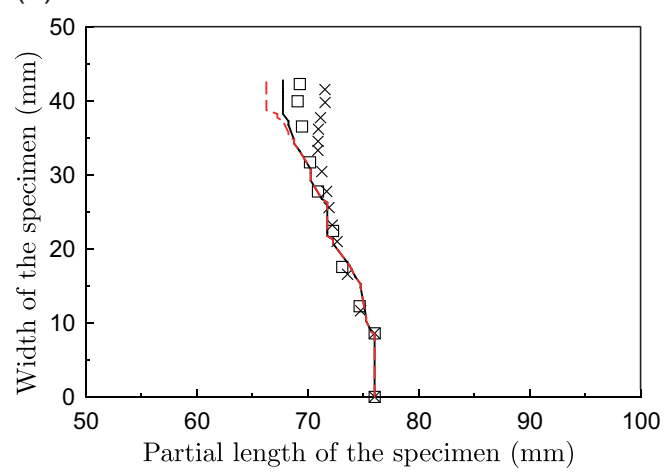

(b)

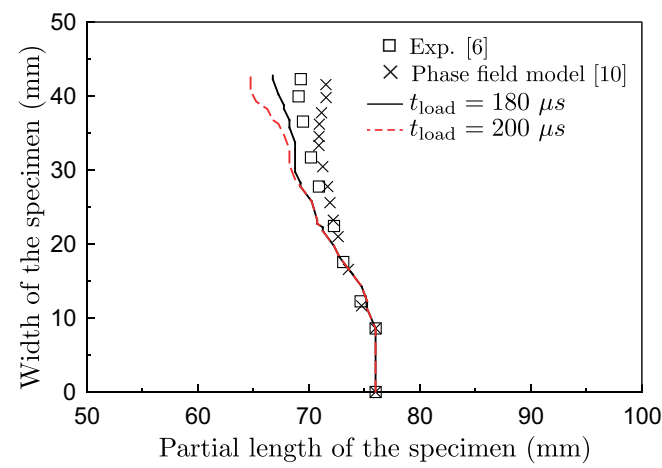

Figure 17: Estimated crack paths for the crack on the stiff side case: (a) constant loading, (b) rump-up-down loading.

In the Fig. 17, the crack path estimations are in good agreement with both the experiment and the phase field model. When the total loading time is $t_{\text {load }}=200 \mu \mathrm{s}$, the crack pattern deviates from the experimental pattern, while the crack patterns become more similar to experimental one in case of $t_{\text {load }}=180 \mu \mathrm{s}$. A small deviation from the experimental crack pattern can be attributed to uncertainty in the loading and boundary conditions between the numerical simulations and the experiments.

It should be noted that the total loading duration does not affect the instance when the crack starts to propagate, but the loading profile will have an obvious effect on the onset of crack propagation. When the constant loading profile is applied on the specimen, the crack starts to propagate much earlier. The crack propagation is observed at $101 \mu \mathrm{s}$ in case of rump-up-down 
loading condition. On the other hand, the constant loading condition triggers crack to propagate at $88.6 \mu \mathrm{s}$. The reported time for the propagation of stiff side crack is $108 \mu \mathrm{s}$ by the bond-based PD [23]. Doan et al. [10] stated that it is difficult to point out exact crack propagation time; however, they provided the variation of bulk and fracture energies. If a special attention is given to energy plot in Ref. [10], it can be observed that the fracture energy starts to increase beyond $100 \mu \mathrm{s}$, which approximately indicates beginning of the crack propagation.

The given results above clearly state that the present OSB-PD modelling estimates the crack pattern and crack propagation time in a good manner. The estimated crack propagation time for the rump-up-down loading profile is in good agreement with the reference bond-based PD results [23], and the estimations by phase field modelling [10].

\subsubsection{Crack on compliant side}

The lower edge, where the crack is located, is assumed to be the compliant side. The variation of material properties is given as:

$$
\begin{gathered}
K_{\mathrm{IC}}(y)=1.4+\frac{2.2-1.4}{43} y, \\
E(y)=4+\frac{10-4}{43} y, \\
\rho(y)=1175+\frac{1750-1175}{43} y .
\end{gathered}
$$

The parameters and units in Eqs. (37)-(39) are same as the crack on stiff side case. The Poisson's ratio is constant as $\nu=0.34$ and the time step size is utilized as $\Delta t=20 \mathrm{~ns}$. The procedure for evaluating critical energy release rates and bond properties is same as the crack on stiff side case.

The obtained crack patterns for the compliant side crack are given in Fig. 18 under different loading profile and durations. It is obvious from the figure that the crack propagation is more like to Mode I (opening mode) as opposed to crack on stiff side cases.

The crack path estimations given in Fig. 18 are consistent with those of Ref. [23]. In addition, it is observed that the crack paths obtained by $t_{\text {load }}=180,200 \mu$ s loading durations coincide well with the experimental results given in Ref. [6]. Accordingly, the experimental crack paths as well as the numerical crack path predictions by Doan et al. [10] were digitized from 


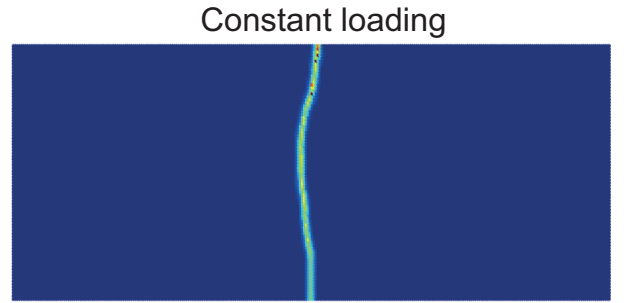

$t_{\mathrm{load}}=100 \mu \mathrm{s}$

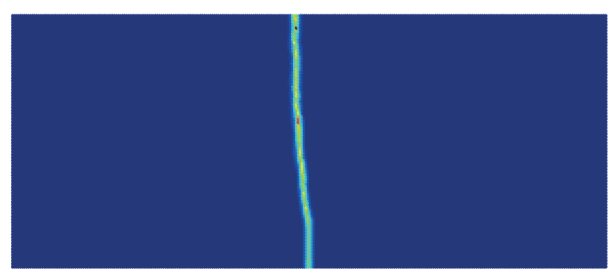

$t_{\text {load }}=150 \mu \mathrm{s}$

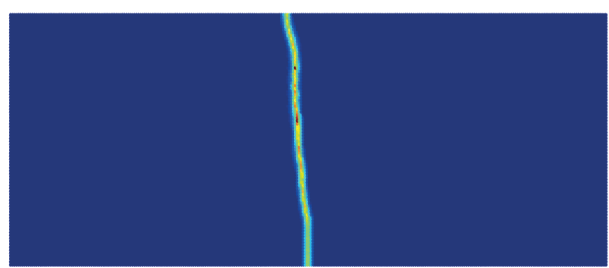

$t_{\text {load }}=180 \mu \mathrm{s}$

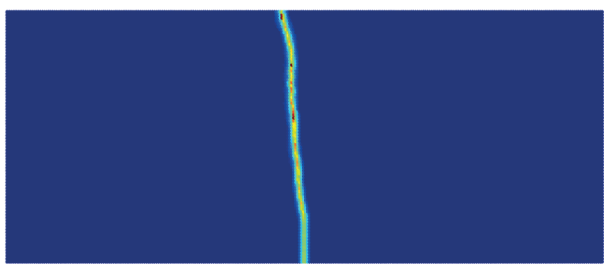

$t_{\text {load }}=200 \mu \mathrm{s}$
Rump-up-down loading
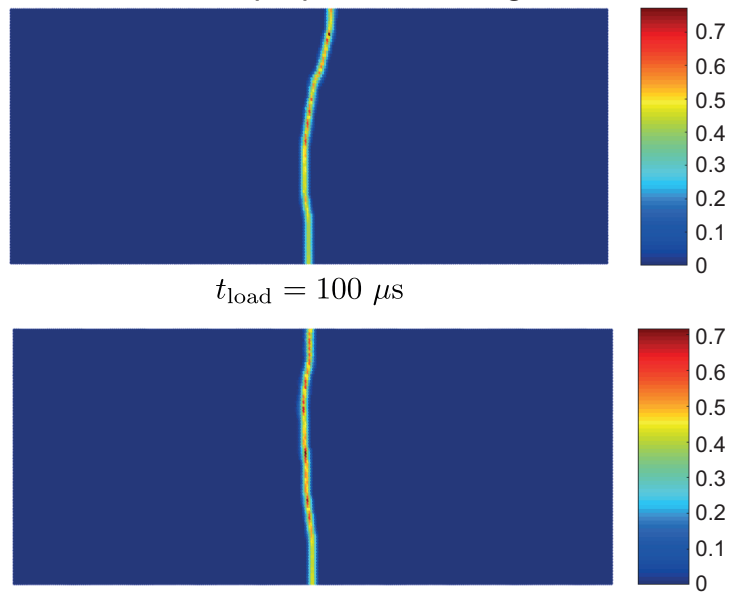

$t_{\text {load }}=150 \mu \mathrm{s}$

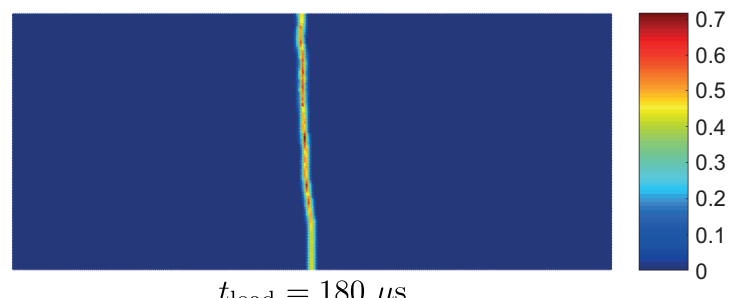

$t_{\text {load }}=180 \mu \mathrm{s}$

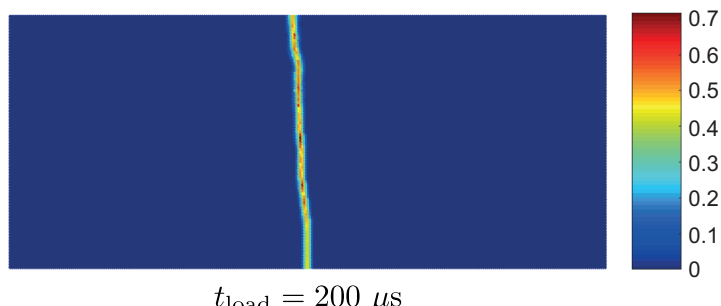

$t_{\text {load }}=200 \mu \mathrm{s}$

Figure 18: Crack patterns of FGMs in which crack is located on compliant side for different loading profiles and durations. 
the figures given in the reference papers for the comparison purposes. These comparisons are presented in Fig. 19. In the given figure, the agreement between crack patterns by the experiment and the present study is much better than that of phase field model. For the compliant side crack, it can be stated that the influence of the loading profile is not much significant compared to that of the loading time. Fig. 19(a) illustrates that the crack patterns for $t_{\text {load }}=180,200 \mu$ s are coincident, while a small deviation between the crack patterns is observed for different total loading durations in case of rump-up-down loading condition, see Fig. 19(b).

In the crack patterns estimated by the present model, a small zigzag behaviour is noticed in Fig. 19, even though the general trend of the crack patterns looks reasonable. This could be explained by the post-processing implementation to detect the crack tip location. As it was pointed out before, the advance of the crack tip is achieved by measuring the local damage parameter. The local damage parameter of material points ahead of the crack tip may exceed 0.5 in a certain time step. At this moment, there are several possibilities for the new crack tip location, which are closely located. It then becomes difficult to track the crack tip precisely, which may lead to a small zig-zag behaviour as observed in Figs. 17 and 19.

(a)

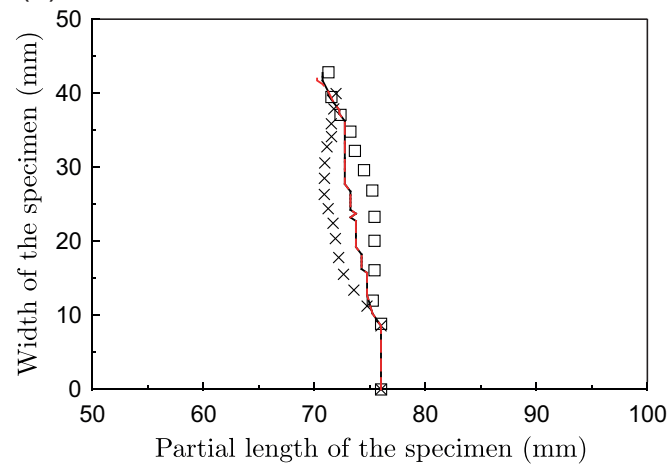

(b)

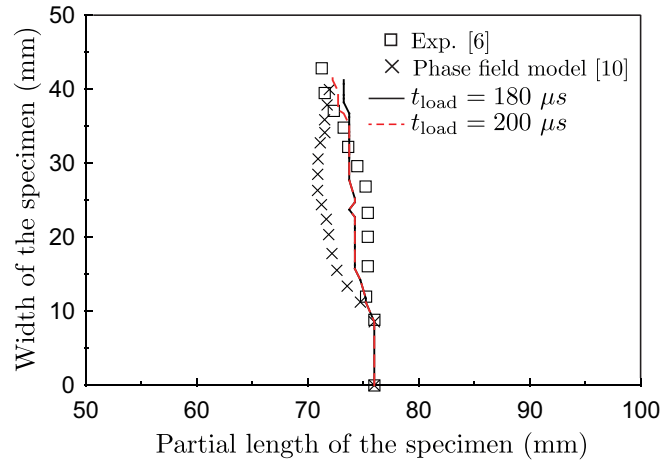

Figure 19: Estimated crack paths for the crack on compliant side case: (a) constant loading, (b) rump-up-down loading.

The onset of crack propagation can be determined as the first instance when the local damage parameter of a material point exceeds 0.5. According to this definition, the crack starts to propagate at $87.4 \mu$ s when constant loading profile is utilized. On the other hand, the crack starts to propagate 
at $104 \mu \mathrm{s}$ for the rump-up-down loading condition. The predicted crack initiation time by the bond-based PD is $125 \mu \mathrm{s}$ [23]. Doan et al. [10] has not provided the exact time, but from the given fracture energy plot, it can be seen that the crack starts to propagate beyond $100 \mu \mathrm{s}$ in Ref. [10]. The crack propagation time by the phase field model is consistent with our prediction.

The deviation between the onset of crack propagation times presented by the reference papers suggest that the crack propagation is influenced by the solution methods employed, loading condition and duration. To sum up, we have achieved good agreement with the results of bond-based PD [23] and phase field model [10].

\section{Conclusions}

The OSB-PD formulation has been implemented for the dynamic fracture analysis of FGMs. Firstly, our computational model was verified by a benchmark problem of crack branching in a homogeneous plate. Good correlation with the reference results is observed. Then, the displacement wave propagation in FGM specimens was simulated under an impact load on the partial length of the top surface. The displacement wave patterns and magnitudes are evaluated precisely in consistent with the reference works.

After verifying our model for the dynamic fracture in a homogeneous plate and simulating the displacement wave propagation, the dynamic fracture problems in FGMs were resolved. The crack branching in FGMs was studied primarily, in which the loading condition is similar to crack branching in the homogeneous plate case. A very good agreement with the reference work is achieved in terms of the crack patterns. The average crack speeds were derived for different division and time step sizes. General trend of the average crack speeds is consistent with each other. The estimated crack speed around the branching instance correlates with the theoretical value in case of fine discretization.

Final numerical example was devoted to the simulation of TPB experiments in FGMs. Two different loading profiles, namely, constant loading and rump-up-down loading were considered. Moreover, the total loading durations were varied to examine the evolution of cracks. The obtained crack patterns were compared with the experimental and numerical works. The estimated crack paths are generally in good agreement with the reference solutions. Onset of the crack propagation instances were examined. Overall, the estimated values of the crack propagation instances are consistent 
with the reference bond-based PD and phase field modelling results for the rump-up-down loading condition.

In the present work, the OSB-PD formulation has been implemented to simulate dynamic fracture of FGMs. Unlike the bond-based PD, present method can represent both dilatational and extensional deformation eliminating the constraints on the material properties. The evaluation of crack propagation is straightforward compared to other numerical methods, e.g., FEM and phase field modelling since calculating the stress-strain field as well as the singular stress field representation are not required. On the other hand, the present model is currently limited to achieve crack propagation by only critical stretch criterion, it is however easy to implement different failure criterion, e.g., energy-based in the OSB-PD framework.

\section{Data availability}

The raw/processed data required to reproduce these findings cannot be shared at this time as the data also forms part of an ongoing study.

\section{Declaration of competing interest}

The authors declare that they have no known competing financial interests or personal relationships that could have appeared to influence the work reported in this paper.

\section{References}

[1] Thai HT, Kim SE. A review of theories for the modeling and analysis of functionally graded plates and shells. Compos Struct 2015;128:70-86.

[2] Berezovski A, Engelbrecht J, Maugin GA. Numerical simulation of twodimensional wave propagation in functionally graded materials. Eur J Mech Solid 2003;22:257-65.

[3] Hedayatrasa S, Bui TQ, Zhang C, Lim CW. Numerical modeling of wave propagation in functionally graded materials using time-domain spectral Chebyshev elements. J Comput Phys 2014;258:381-404.

[4] Eischen JW. Fracture of nonhomogeneous materials. Int J Fract $1987 ; 34: 3-22$. 
[5] Abanto-Bueno J, Lambros J. An experimental study of mixed mode crack initiation and growth in functionally graded materials. Exp Mech 2006;46:179-96.

[6] Kirugulige MS, Tippur HV. Mixed-mode dynamic crack growth in functionally graded glass-filled epoxy. Exp Mech 2006;46:269-81.

[7] Kirugulige M, Tippur HV. Mixed-mode dynamic crack growth in a functionally graded particulate composite: experimental measurements and finite element simulations. J Appl Mech 2008;0511021.

[8] Kidane A, Chalivendra VB, Arun S, Chona R. Mixed-mode dynamic crack propagation in graded materials under thermo-mechanical loading. Eng Fract Mech 2010;77:2864-80.

[9] Abotula S, Kidane A, Chalivendra VB, Shukla A. Dynamic curving cracks in functionally graded materials under thermo-mechanical loading. Int J Solid Struct 2012;49:1637-55.

[10] Doan DH, Bui TQ, Duc ND, Fushinobu K. Hybrid phase field simulation of dynamic crack propagation in functionally graded glass-filled epoxy. Compos B Eng 2016;99:266-76.

[11] Hirshikesh, Sundararajan N, Ratna KA, Emilio MP. Phase field modelling of crack propagation in functionally graded materials. Composites Part B: Engineering 2019;169:139-48.

[12] Silling SA. Reformulation of elasticity theory for discontinuities and long-range forces. Journal of the Mechanics and Physics of Solids 2000;48:175-209.

[13] Silling SA, Askari E. A meshfree method based on the peridynamic model of solid mechanics. Comput Struct 2005;83:1526-35.

[14] Silling SA, Epton M, Weckner O, Xu J, Askari E. Peridynamic states and constitutive modeling. J Elasticity 2007;88:151-84.

[15] Ha YD, Bobaru F. Studies of dynamic crack propagation and crack branching with peridynamics. Int J Fract 2010;162:229-44.

[16] Ha YD, Bobaru F. Characteristics of dynamic brittle fracture captured with peridynamics. Eng Fract Mech 2011;78:1156-68. 
[17] Madenci E, Colavito K, Phan N. Peridynamics for unguided crack growth prediction under mixed-mode loading. Eng Fract Mech 2016;167:34-44.

[18] Dipasquale D, Sarego G, Zaccariotto M, Galvanetto U. A discussion on failure criteria for ordinary state-based peridynamics. Eng Fract Mech 2017;186:378-98.

[19] Imachi M, Tanaka S, Bui TQ. Mixed-mode dynamic stress intensity factors evaluation using ordinary state-based peridynamics. Theor Appl Fract Mech 2018;93:97-104.

[20] Imachi M, Tanaka S, Bui TQ, Oterkus S, Oterkus E. A computational approach based on ordinary state-based peridynamics with new transition bond for dynamic fracture analysis. Eng Fract Mech 2019;206:35974 .

[21] Imachi M, Tanaka S, Ozdemir M, Bui TQ, Oterkus S, Oterkus E. Dynamic crack arrest analysis by ordinary state-based peridynamics. Int J Fract 2020;221:155-169.

[22] Basoglu MF, Zerin Z, Kefal A, Oterkus E. A computational model of peridynamic theory for deflecting behavior of crack propagation with micro-cracks. Comput Mater Sci 2019;162:33-46.

[23] Cheng Z, Zhang G, Wang Y, Bobaru F. A peridynamic model for dynamic fracture in functionally graded materials. Compos Struct 2015;133:529-46.

[24] Cheng Z, Liu Y, Zhao J, Feng H, Wu Y. Numerical simulation of crack propagation and branching in functionally graded materials using peridynamic modeling. Eng Fract Mech 2018;191:13-32.

[25] Cheng Z, Sui Z, Yin H, Feng H. Numerical simulation of dynamic fracture in functionally graded materials using peridynamic modeling with composite weighted bonds. Eng Anal Bound Elem 2019;105:31-46.

[26] Cheng ZQ, Sui ZB, Yin H, Yuan CF, Chu LS. Studies of dynamic fracture in functionally graded materials using peridynamic modeling with composite weighted bond. Theor Appl Fract Mech 2019;103:102242. 
[27] Liao Y, Liu L, Liu Q, Lai X, Assefa M, Liu J. Peridynamic simulation of transient heat conduction problems in functionally gradient materials with cracks. J Therm Stresses 2017;40:1484-501.

[28] Madenci E, Oterkus E. Peridynamic Theory and Its Applications. USA: Springer; 2013.

[29] Javili A, Morasata R, Oterkus E, Oterkus S. Peridynamics review. Math Mech Solid 2018;24:3714-39.

[30] Kilic B, Agwai A, Madenci E. Peridynamic theory for progressive damage prediction in center-cracked composite laminates. Compos Struct 2009;90:141-51.

[31] Oterkus E, Madenci E. Peridynamic analysis of fiber-reinforced composite materials. J Mech Mater Struct 2012;6:45-84.

[32] Hu YL, De Carvalho NV, Madenci E. Peridynamic modeling of delamination growth in composite laminates. Compos Struct 2015;132:610-20.

[33] Diyaroglu C, Oterkus E, Madenci E, Rabczuk T, Siddiq A. Peridynamic modeling of composite laminates under explosive loading. Compos Struct 2016;144:14-23.

[34] Le QV, Chan WK, Schwartz J. A two-dimensional ordinary, state-based peridynamic model for linearly elastic solids. Int J Numer Meth Eng 2014;27:148-53.

[35] Bowden FP, Brunton JH, Field JE, Heyes AD. Controlled fracture of brittle solids and interruption of electrical current. Nature 1967;216:3842 .

[36] Ravi-Chandar K. Dynamic fracture. USA: Elsevier; 2004. 